

\title{
Affine congruences and rational points on a certain cubic surface
}

\author{
Pierre Le Boudec
}

We establish estimates for the number of solutions of certain affine congruences. These estimates are then used to prove Manin's conjecture for a cubic surface split over $\mathbb{Q}$ whose singularity type is $\mathrm{D}_{4}$. This improves on a result of Browning and answers a problem posed by Tschinkel.

1. Introduction

2. Preliminaries

1263

3. The universal torsor

1277

4. Calculation of Peyre's constant

1278

5. Proof of the main theorem

1279

Acknowledgments

1294

References

1294

\section{Introduction}

The aim of this paper is to study the asymptotic behavior of the number of rational points of bounded height on the cubic surface $V \subset \mathbb{P}^{3}$ defined over $\mathbb{Q}$ by

$$
x_{0}\left(x_{1}+x_{2}+x_{3}\right)^{2}-x_{1} x_{2} x_{3}=0 .
$$

Manin's conjecture [Franke et al. 1989], and the refinements concerning the value of the constant due to Peyre [1995] and to Batyrev and Tschinkel [1998b], describe precisely what should be the solution of this problem.

The variety $V$ has a unique singularity at the point $(1: 0: 0: 0)$, of type $\mathrm{D}_{4}$. In addition, it contains precisely six lines, which are defined by $x_{0}=x_{i}=0$ and $x_{1}+x_{2}+x_{3}=x_{i}=0$ for $i \in\{1,2,3\}$. Rational points accumulate on these six lines, hiding the interesting behavior of the number of rational points lying outside the lines. We thus let $U$ be the open subset formed by removing the six lines

MSC2010: primary 11D45; secondary 14G05.

Keywords: affine congruences, rational points, Manin's conjecture, cubic surfaces, universal torsors. 
from $V$. We also let $H: \mathbb{P}^{3}(\mathbb{Q}) \rightarrow \mathbb{R}_{>0}$ be the exponential height, defined for a vector $\left(x_{0}, x_{1}, x_{2}, x_{3}\right) \in \mathbb{Z}^{4}$ satisfying $\operatorname{gcd}\left(x_{0}, x_{1}, x_{2}, x_{3}\right)=1$ by

$$
H\left(x_{0}: x_{1}: x_{2}: x_{3}\right)=\max \left\{\left|x_{0}\right|,\left|x_{1}\right|,\left|x_{2}\right|,\left|x_{3}\right|\right\} .
$$

The quantity in which we are interested is then defined by

$$
N_{U, H}(B)=\#\{x \in U(\mathbb{Q}) \mid H(x) \leq B\} .
$$

In this specific context, Manin's conjecture states that

$$
N_{U, H}(B)=c_{V, H} B(\log B)^{6}(1+o(1)),
$$

where $c_{V, H}$ is a constant which is expected to agree with Peyre's prediction. In a more general setting, the exponent of the logarithm is expected to be equal to the rank of the Picard group of the minimal desingularization of $V$ minus one. In comparison, the number $N_{\mathbb{P}^{1}, H}(B)$ of rational points of bounded height lying on a line satisfies $N_{\mathbb{P}^{1}, H}(B)=c_{\mathbb{P}^{1}, H} B^{2}(1+o(1))$, where $c_{\mathbb{P}^{1}, H}>0$.

Manin's conjecture for singular cubic surfaces has received an increasing amount of attention over the last years (see, for instance, [de la Bretèche and SwinnertonDyer 2007; de la Bretèche et al. 2007; Le Boudec 2012a]). The interested reader is invited to refer to [Le Boudec 2012a, Section 1] for a comprehensive overview of what is currently known concerning singular cubic surfaces defined over $\mathbb{Q}$.

Any cubic surface in $\mathbb{P}^{3}$ defined over $\mathbb{C}$ which has only isolated singularities and which is not a cone over an elliptic curve can only have ADE singularities (see [Coray and Tsfasman 1988, Proposition 0.2]). In Table 1 below, we recall the classification over $\overline{\mathbb{Q}}$ of cubic surfaces with ADE singularities, and we give the number of lines contained by the surfaces. Moreover, we indicate if Manin's conjecture is known for at least one example of the surface of the specified singularity type by giving the corresponding reference. Note that the difficulty of proving Manin's conjecture increases as we go higher in Table 1.

At the American Institute of Mathematics workshop Rational and integral points on higher-dimensional varieties in 2002, Tschinkel posed the problem of studying the quantity $N_{U, H}(B)$. Motivated by [Heath-Brown 2003], which deals with Cayley's cubic surface, Browning [2006] gave a first answer to this question by proving that

$$
N_{U, H}(B) \asymp B(\log B)^{6},
$$

where $\asymp$ means that the ratio of these two quantities is between two positive constants. To do so, he made use of the universal torsor calculated in [Hassett and Tschinkel 2004], which is an open subset of the affine hypersurface embedded in $\mathbb{A}^{10} \simeq \operatorname{Spec}\left(\mathbb{Q}\left[\eta_{1}, \ldots, \eta_{10}\right]\right)$ and defined by

$$
\eta_{2} \eta_{5}^{2} \eta_{8}+\eta_{3} \eta_{6}^{2} \eta_{9}+\eta_{4} \eta_{7}^{2} \eta_{10}-\eta_{1} \eta_{2} \eta_{3} \eta_{4} \eta_{5} \eta_{6} \eta_{7}=0 .
$$




\begin{tabular}{rrr} 
Singularity type & Number of lines & Result \\
\hline $\mathrm{A}_{1}$ & 21 & \\
$2 \mathrm{~A}_{1}$ & 16 & \\
$\mathrm{~A}_{2}$ & 15 & \\
$3 \mathrm{~A}_{1}$ & 12 & \\
$\mathrm{~A}_{2}+\mathrm{A}_{1}$ & 11 & \\
$\mathrm{~A}_{3}$ & 10 & \\
$4 \mathrm{~A}_{1}$ & 9 & \\
$2 \mathrm{~A}_{1}+\mathrm{A}_{2}$ & 8 & \\
$\mathrm{~A}_{3}+\mathrm{A}_{1}$ & 7 & \\
$2 \mathrm{~A}_{2}$ & 7 & \\
$\mathrm{~A}_{4}$ & 6 & \\
$\mathrm{D}_{4}$ & 6 & \\
$2 \mathrm{~A}_{1}+\mathrm{A}_{3}$ & 5 & \\
$2 \mathrm{~A}_{2}+\mathrm{A}_{1}$ & 5 & \\
$\mathrm{~A}_{4}+\mathrm{A}_{1}$ & 4 & \\
$\mathrm{~A}_{5}$ & 3 & \\
$\mathrm{D}_{5}$ & 3 & [Brownis paper] \\
$3 \mathrm{~A}_{2}$ & 3 & [Batyrev and Tschinkel 1998a] \\
$\mathrm{A}_{5}+\mathrm{A}_{1}$ & 2 & [Baier and Derenthal 2012] \\
$\mathrm{E}_{6}$ & 1 & [de la Bretèche et al. 2007]
\end{tabular}

Table 1. Cubic surfaces with ADE singularities.

In this paper, we also make use of this auxiliary variety to establish Manin's conjecture for $V$.

Universal torsors were originally introduced by Colliot-Thélène and Sansuc in order to study the Hasse principle and weak approximation for rational varieties (see [Colliot-Thélène and Sansuc 1976; 1980; 1987]). These descent methods have turned out to be a very pertinent tool for counting problems. The parametrizations of rational points provided by universal torsors have been used in the context of Manin's conjecture for the first time by Peyre [1998] and Salberger [1998].

It is a well-established heuristic that counting rational points on cubic surfaces becomes harder as the number $N$ of $(-2)$-curves on the minimal desingularizations decreases (which means as we go higher in Table 1). As a consequence, our result can be seen as a new record, since $V$ is the first example of cubic surface with $N=4$ for which Manin's conjecture is proved. By way of comparison, we record 
here that $N$ is also equal to 4 for Cayley's cubic. Previously, Manin's conjecture was known for only two nontoric cubic surfaces with $N=6$ (see [de la Bretèche et al. 2007; Baier and Derenthal 2012]) and two cubic surfaces with $N=5$ (see [Browning and Derenthal 2009; Le Boudec 2012a]).

Since the parametrizations of the rational points resorting to universal torsors become extremely complicated as $N$ decreases, it seems to the author that establishing Manin's conjecture for a cubic surface with $1 \leq N \leq 3$, and even for another cubic surface with $N=4$, is an extremely difficult problem. In particular, all such surfaces have universal torsors which are not hypersurfaces. Actually, it is not even clear if sharp upper bounds for $N_{U, H}(B)$ can be obtained for surfaces with $1 \leq N \leq 3$. As a reminder, the best result known for nonsingular cubic surfaces (that is, with $N=0$ ) is the upper bound

$$
N_{U, H}(B) \ll B^{4 / 3+\varepsilon}
$$

for any fixed $\varepsilon>0$, which holds if the surface contains three coplanar lines defined over $\mathbb{Q}$ (see [Heath-Brown 1997]).

To prove Manin's conjecture for $V$, we start by establishing estimates for the number of $(u, v) \in \mathbb{Z}^{2}$ lying in a prescribed region and satisfying the congruence

$$
a_{1} u+a_{2} v \equiv b(\bmod q)
$$

and the condition $\operatorname{gcd}(u v, q)=1$, where $a_{1}, a_{2} \in \mathbb{Z}_{\neq 0}, q \in \mathbb{Z}_{\geq 1}$ are such that $a_{1} a_{2}$ is coprime to $q$ and $b \in \mathbb{Z}$ is divisible by each prime number dividing $q$. Then, the first step of the proof consists in summing over three variables, viewing the torsor equation as an affine congruence to which these estimates are applied.

At this stage of the proof, a very interesting phenomenon stands out. The error term showing up in these estimates gives birth to a new congruence where the coefficients $a_{1}$ and $a_{2}$ appear. However, it is not possible to give a good bound for this quantity for any fixed $a_{1}$ and $a_{2}$ coprime to $q$. As a consequence, this quantity has to be estimated on average over certain variables dividing $a_{1}$ and $a_{2}$. More precisely, this error term is nontrivially summed over two other variables whose squares respectively divide $a_{1}$ and $a_{2}$, using a result due to Heath-Brown and coming from the geometry of numbers.

The step which makes this new congruence appear is definitely the key step of our proof (see Lemma 2). Our method is believed to be quite new and will certainly be useful in dealing with other diophantine problems. For instance, the methods of Lemmas 2 and 9 are used in forthcoming work of la Bretèche and Browning [2014], in which they study in a quantitative way the failure of the Hasse principle for a certain family of Châtelet surfaces.

It is worth pointing out that it is very likely that our work can be adapted to yield a proof of Manin's conjecture for another cubic surface with a single singularity 
of type $\mathrm{D}_{4}$ but lying in the other isomorphism class over $\overline{\mathbb{Q}}$ (there are exactly two isomorphism classes of cubic surfaces with $\mathrm{D}_{4}$ singularity type over $\overline{\mathbb{Q}}$ ). This cubic surface is defined over $\mathbb{Q}$ by

$$
x_{0}\left(x_{1}+x_{2}+x_{3}\right)^{2}+x_{1}\left(x_{1}+x_{2}\right)=0,
$$

and the universal torsor corresponding to this problem is an open subset of the affine hypersurface embedded in $\mathbb{A}^{10} \simeq \operatorname{Spec}\left(\mathbb{Q}\left[\eta_{1}, \ldots, \eta_{10}\right]\right)$ and defined by

$$
\eta_{2} \eta_{5}^{2} \eta_{8}+\eta_{3} \eta_{6}^{2} \eta_{9}+\eta_{4} \eta_{7}^{2} \eta_{10}=0
$$

The study of the congruence (1-1) in the particular case $b=0$ is expected to solve the problem of proving Manin's conjecture for this surface in a similar fashion.

Our main result is the following:

Theorem 1. As $B$ tends to $+\infty$, we have the estimate

$$
N_{U, H}(B)=c_{V, H} B(\log B)^{6}\left(1+O\left(\frac{1}{(\log \log B)^{1 / 6}}\right)\right),
$$

where $c_{V, H}$ agrees with Peyre's prediction.

It has been checked that $V$ is not an equivariant compactification of $\mathbb{G}_{m}^{2}$ or $\mathbb{G}_{a}^{2}$ (see [Derenthal 2014, Proposition 13] and [Derenthal and Loughran 2010]). Furthermore, let

$$
G_{d}=\mathbb{G}_{a} \rtimes_{d} \mathbb{G}_{m},
$$

where $d \in \mathbb{Z}$ and the action of $g \in \mathbb{G}_{m}$ on $x \in \mathbb{G}_{a}$ is given by $g \cdot x=g^{d} x$. It can be checked that if $V$ were an equivariant compactification of $G_{d}$, then the number of negative curves on its minimal desingularization would be less than or equal to 8 , which is not the case since this number is equal to 10 . As a result, Theorem 1 does not follow from the general results concerning equivariant compactifications of algebraic groups [Batyrev and Tschinkel 1998a; Chambert-Loir and Tschinkel 2002; Tanimoto and Tschinkel 2012].

The next section is dedicated to the proofs of several preliminary results. The two following sections are devoted to the descriptions of the universal torsor and Peyre's constant respectively. Finally, in the remaining section we prove Theorem 1.

Throughout the proof, $\varepsilon$ is an arbitrarily small positive number. As a convention, the implicit constants involved in the notation $O$ and $\ll$ are always allowed to depend on $\varepsilon$.

\section{Preliminaries}

2.1. Affine congruences. Let $a_{1}, a_{2} \in \mathbb{Z}_{\neq 0}$ be two integers, and set $\boldsymbol{a}=\left(a_{1}, a_{2}\right)$. Let also $q \in \mathbb{Z}_{\geq 1}$ and $b \in \mathbb{Z}$. We assume that $a_{1} a_{2}$ is coprime to $q$. Moreover, if we let $\operatorname{rad}(n)$ denote the radical of an integer $n \geq 1$; that is, 


$$
\operatorname{rad}(n)=\prod_{p \mid n} p
$$

then we also assume that

$$
\operatorname{rad}(q) \mid b
$$

Let $\Phi$ and $\Phi$ be two bounded intervals. We introduce the quantities

$$
\begin{aligned}
& N(\mathscr{I}, \mathscr{I} ; q, \boldsymbol{a}, b) \\
& \quad=\#\left\{(u, v) \in \mathscr{I} \times \mathscr{S} \cap \mathbb{Z}^{2} \mid a_{1} u+a_{2} v \equiv b(\bmod q), \operatorname{gcd}(u v, q)=1\right\},
\end{aligned}
$$

and

$$
N^{*}(\mathscr{I}, \mathscr{g} ; q)=\frac{1}{\varphi(q)} \#\left\{(u, v) \in \mathscr{I} \times \mathscr{\mathscr { S }} \cap \mathbb{Z}^{2} \mid \operatorname{gcd}(u v, q)=1\right\} .
$$

It is immediate to check that one of the two conditions among $\operatorname{gcd}(u, q)=1$ and $\operatorname{gcd}(v, q)=1$ can be omitted in the definition of $N(\mathscr{I}, \mathscr{F} ; q, \boldsymbol{a}, b)$. Indeed, if we omit the condition $\operatorname{gcd}(u, q)=1$, then the conditions $\operatorname{gcd}\left(a_{2}, q\right)=1$ and $\operatorname{gcd}(v, q)=1$ together imply that we have $\operatorname{gcd}\left(a_{1} u-b, q\right)=1$. This last condition is seen to be equivalent to $\operatorname{gcd}(u, q)=1$, thanks to the conditions $(2-1)$ and $\operatorname{gcd}\left(a_{1}, q\right)=1$.

Note that $N^{*}(\mathscr{I}, \mathscr{F} ; q)$ is the average of $N(\mathscr{I}, \mathscr{F} ; q, \boldsymbol{a}, b)$ over $a_{1}$ or $a_{2}$ coprime to $q$. In Lemma 2 , we show how we can approximate $N(\mathscr{I}, \mathscr{F} ; q, \boldsymbol{a}, b)$ by $N^{*}(\mathscr{I}, \mathscr{F} ; q)$. We start by studying some exponential sums which will naturally appear in the proof of Lemma 2. For $q \in \mathbb{Z}_{\geq 1}$, we let $e_{q}$ be the function defined by $e_{q}(x)=e^{2 i \pi x / q}$, and we set for $r, s \in \mathbb{Z}$

$$
S_{q}(r, s, \boldsymbol{a}, b)=\sum_{\substack{\alpha, \beta=1 \\ \operatorname{gcd}(\alpha \beta, q)=1 \\ a_{1} \alpha+a_{2} \beta \equiv b(\bmod q)}}^{q} e_{q}(r \alpha+s \beta) .
$$

Furthermore, we need to introduce the classical Ramanujan sum. For $q \in \mathbb{Z}_{\geq 1}$ and $n \in \mathbb{Z}$, we set

$$
c_{q}(n)=\sum_{\substack{\alpha=1 \\ \operatorname{gcd}(\alpha, q)=1}}^{q} e_{q}(n \alpha)
$$

and we recall that

$$
c_{q}(n)=\sum_{d \mid \operatorname{gcd}(q, n)} \mu\left(\frac{q}{d}\right) d .
$$

Lemma 1. For any $r, s \in \mathbb{Z}$, we have

$$
S_{q}(r, s, \boldsymbol{a}, b)=e_{q}\left(r a_{1}^{-1} b\right) c_{q}\left(a_{1} s-a_{2} r\right)
$$

and, symmetrically,

$$
S_{q}(r, s, \boldsymbol{a}, b)=e_{q}\left(s a_{2}^{-1} b\right) c_{q}\left(a_{2} r-a_{1} s\right),
$$


where $a_{1}^{-1}$ and $a_{2}^{-1}$ denote respectively the inverses of $a_{1}$ and $a_{2}$ modulo $q$.

As a result, we have $S_{q}(q, s, \boldsymbol{a}, b)=c_{q}(s)$ and $S_{q}(r, q, \boldsymbol{a}, b)=c_{q}(r)$, and thus these two quantities are independent of $\boldsymbol{a}$ and $b$.

Proof. The symmetry given by the map $\left(r, s, a_{1}, a_{2}\right) \mapsto\left(s, r, a_{2}, a_{1}\right)$ implies that we only need to prove one of the two equalities. Let us prove the second one. Just as we can omit the condition $\operatorname{gcd}(v, q)=1$ in the definition of $N(\mathscr{g}, \mathscr{g} ; q, \boldsymbol{a}, b)$, we can also omit the condition $\operatorname{gcd}(\beta, q)=1$ in the definition of $S_{q}(r, s, \boldsymbol{a}, b)$. Therefore, we get

$$
\begin{aligned}
S_{q}(r, s, \boldsymbol{a}, b) & =\sum_{\substack{\alpha=1 \\
\operatorname{gcd}(\alpha, q)=1}}^{q} e_{q}(r \alpha) \sum_{\substack{\beta=1 \\
a_{1} \alpha+a_{2} \beta \equiv b(\bmod q)}}^{q} e_{q}(s \beta) \\
& =\sum_{\substack{\alpha=1 \\
\operatorname{gcd}(\alpha, q)=1}}^{q} e_{q}(r \alpha) e_{q}\left(s\left(a_{2}^{-1} b-a_{2}^{-1} a_{1} \alpha\right)\right) \\
& =e_{q}\left(s a_{2}^{-1} b\right) \sum_{\substack{\alpha=1 \\
\operatorname{gcd}(\alpha, q)=1}}^{q} e_{q}\left(\left(r-a_{2}^{-1} a_{1} s\right) \alpha\right) \\
& =e_{q}\left(s a_{2}^{-1} b\right) c_{q}\left(r-a_{2}^{-1} a_{1} s\right)=e_{q}\left(s a_{2}^{-1} b\right) c_{q}\left(a_{2} r-a_{1} s\right),
\end{aligned}
$$

as wished.

From now on, for $\lambda>0$ we define the arithmetic function $\sigma_{-\lambda}$ by

$$
\sigma_{-\lambda}(n)=\sum_{k \mid n} k^{-\lambda}
$$

Lemma 2. Let $a_{1}, a_{2} \in \mathbb{Z}_{\neq 0}, q \in \mathbb{Z}_{\geq 1}$ and $b \in \mathbb{Z}$, satisfying the assumptions $\operatorname{gcd}\left(a_{1} a_{2}, q\right)=1$ and $\operatorname{rad}(q) \mid b$. We have the estimate

$$
N(\mathscr{I}, \mathscr{g} ; q, \boldsymbol{a}, b)-N^{*}(\mathscr{I}, \mathscr{g} ; q) \ll E(q, \boldsymbol{a}),
$$

where $E(q, \boldsymbol{a})=E_{0}(q, \boldsymbol{a})+E_{1}(q)$ with

$$
E_{0}(q, \boldsymbol{a})=\sum_{d \mid q}\left|\mu\left(\frac{q}{d}\right)\right| d \sum_{\substack{0<|r|,|s| \leq q / 2 \\ a_{1} s-a_{2} r \equiv 0(\bmod d)}}|r|^{-1}|s|^{-1}
$$

and

$$
E_{1}(q)=\left(\frac{q}{\varphi(q)}\right)^{3}(\log q)^{2}
$$


Proof. We detect the congruence using sums of exponentials; we get

$$
\begin{aligned}
N(\mathscr{I}, \mathscr{g} ; q, \boldsymbol{a}, b)= & \sum_{\substack{\alpha, \beta=1 \\
\operatorname{ccd}(\alpha \beta, q)=1 \\
a_{1} \alpha+a_{2} \beta \equiv b(\bmod q)}}^{q} \#\left\{(u, v) \in \mathscr{I} \times \mathscr{J} \cap \mathbb{Z}^{2}|q| \alpha-u, \beta-v\right\} \\
= & \sum_{\substack{\alpha, \beta=1 \\
\operatorname{gcd}(\alpha \beta, q)=1 \\
a_{1} \alpha+a_{2} \beta \equiv b(\bmod q)}}^{q} \frac{1}{q^{2}}\left(\sum_{u \in \mathscr{I}} \sum_{r=1}^{q} e_{q}(r \alpha-r u)\right)\left(\sum_{v \in \mathscr{I}} \sum_{s=1}^{q} e_{q}(s \beta-s v)\right) \\
= & \frac{1}{q^{2}} \sum_{r, s=1}^{q} S_{q}(r, s, \boldsymbol{a}, b) F_{q}(r, s),
\end{aligned}
$$

where

$$
F_{q}(r, s)=\left(\sum_{u \in \mathscr{I}} e_{q}(-r u)\right)\left(\sum_{v \in \mathscr{F}} e_{q}(-s v)\right) .
$$

Using Lemma 1, we get

$$
N(\Phi, \mathscr{g} ; q, \boldsymbol{a}, b)=\frac{1}{q^{2}} \sum_{r, s=1}^{q} e_{q}\left(r a_{1}^{-1} b\right) c_{q}\left(a_{1} s-a_{2} r\right) F_{q}(r, s) .
$$

Let $\|x\|$ denote the distance from $x$ to the set of integers. If $r, s \neq q$, then $F_{q}(r, s)$ is the product of two geometric sums, and we therefore have

$$
F_{q}(r, s) \ll\left\|\frac{r}{q}\right\|^{-1}\left\|\frac{s}{q}\right\|^{-1} .
$$

Let $N(\mathscr{g}, \mathscr{g} ; q)$ be the sum of the terms corresponding to $r=q$ or $s=q$. As stated in Lemma $1, N(\Phi, \Phi ; q)$ is independent of $a_{1}, a_{2}$ and $b$. Using (2-4), we get

$$
\begin{aligned}
N(\mathscr{I}, \mathscr{F} ; q, \boldsymbol{a}, b)-N(\mathscr{I}, \mathscr{g} ; q) & =\frac{1}{q^{2}} \sum_{r, s=1}^{q-1} e_{q}\left(r a_{1}^{-1} b\right) c_{q}\left(a_{1} s-a_{2} r\right) F_{q}(r, s) \\
& \ll \frac{1}{q^{2}} \sum_{d \mid q}\left|\mu\left(\frac{q}{d}\right)\right| d \sum_{\substack{r, s=1 \\
a_{1} s-a_{2} r=0(\bmod d)}}^{q-1}\left\|\frac{r}{q}\right\|^{-1}\left\|\frac{s}{q}\right\|^{-1} \\
& \ll \frac{1}{q^{2}} \sum_{d \mid q}\left|\mu\left(\frac{q}{d}\right)\right| d \sum_{\substack{0<|r|,|s| \leq q / 2 \\
a_{1} s-a_{2} r=0(\bmod d)}} \frac{q}{|r|} \frac{q}{|s|} .
\end{aligned}
$$

Recall that the right-hand side is equal to $E_{0}(q, \boldsymbol{a})$. We have thus obtained

$$
N(\mathscr{I}, \mathscr{F} ; q, \boldsymbol{a}, b)-N(\mathscr{I}, \mathscr{g} ; q) \ll E_{0}(q, \boldsymbol{a}) .
$$


Since $N(\mathscr{I}, \mathscr{F} ; q)$ is independent of $a_{2}$ and since $N^{*}(\mathscr{I}, \mathscr{F} ; q)$ is the average of $N(\mathscr{I}, \mathscr{g} ; q, \boldsymbol{a}, b)$ over $a_{2}$ coprime to $q$, averaging this estimate over $a_{2}$ coprime to $q$ shows that

$$
N^{*}(\mathscr{I}, \mathscr{g} ; q)-N(\mathscr{I}, \mathscr{g} ; q) \ll E_{1}^{\prime}(q)
$$

where

$$
\begin{aligned}
E_{1}^{\prime}(q) & =\frac{1}{\varphi(q)} \sum_{d \mid q} d \sum_{0<|r|,|s| \leq q / 2}|r|^{-1}|s|^{-1} \sum_{\substack{a_{2}=1 \\
\operatorname{gcd}\left(a_{2}, q\right)=1 \\
a_{1} s-a_{2} r=0(\bmod d)}}^{q} 1 \\
& \ll \frac{1}{\varphi(q)} \sum_{d \mid q} d \sum_{0<|r|,|s| \leq q / 2} \operatorname{gcd}(r, s, d)|r|^{-1}|s|^{-1} \\
& \ll \frac{1}{\varphi(q)} \sum_{d \mid q} d \sum_{d^{\prime} \mid d} d^{\prime} \sum_{0<|r|,|s| \leq q / 2}|r|^{-1}|s|^{-1} \\
& \ll \frac{1}{\varphi(q)}(\log q)^{2} \sum_{d\left|r, d^{\prime}\right| s} d \sigma_{-1}(d) .
\end{aligned}
$$

Furthermore, we can check that the right-hand side is bounded by $E_{1}(q)$. Thus

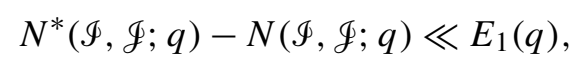

and therefore, combining the estimates (2-5) and (2-6), we obtain

$$
N(\mathscr{I}, \mathscr{q} ; q, \boldsymbol{a}, b)-N^{*}(\mathscr{I}, \mathscr{F} ; q) \ll E(q, \boldsymbol{a}),
$$

which completes the proof.

Note that an immediate consequence of Lemma 2 is the bound

$$
N(\mathscr{I}, \mathscr{g} ; q, \boldsymbol{a}, b) \ll \frac{1}{\varphi(q)} \#\left(\mathscr{I} \times \mathscr{F} \cap \mathbb{Z}^{2}\right)+E(q, \boldsymbol{a}) .
$$

We now introduce a certain domain $\mathscr{Y} \subset \mathbb{R}^{2}$ where the couple $(u, v)$ is restricted to lie. Let $X, T, A_{1}, A_{2} \geq 1$. We let $\mathscr{Y}=\mathscr{Y}\left(X, T, A_{1}, A_{2}\right)$ be the set of $(x, y) \in \mathbb{R}^{2}$ such that

$$
\begin{aligned}
A_{1}|x| A_{2}|y|\left|A_{1} x+A_{2} y-T\right| & \leq T^{2} X, \\
\left|A_{1} x+A_{2} y-T\right| & \leq X, \\
A_{1}|x| & \leq X, \\
A_{2}|y| & \leq X .
\end{aligned}
$$

Note that the last three conditions imply that we also have

$$
T \leq 3 X \text {. }
$$


Finally, we set

$$
D(\mathscr{Y} ; q, \boldsymbol{a}, b)=\#\left\{(u, v) \in \mathscr{S} \cap \mathbb{Z}_{\neq 0}^{2} \mid a_{1} u+a_{2} v \equiv b(\bmod q), \operatorname{gcd}(u v, q)=1\right\}
$$

and

$$
D^{*}(\mathscr{Y} ; q)=\frac{1}{\varphi(q)} \#\left\{(u, v) \in \mathscr{Y} \cap \mathbb{Z}_{\neq 0}^{2} \mid \operatorname{gcd}(u v, q)=1\right\} .
$$

We now aim to prove the following lemma.

Lemma 3. Let $L \geq 1$. We have the estimate

$$
D(\varphi ; q, \boldsymbol{a}, b)-D^{*}(\varphi ; q) \ll \frac{1}{L} \frac{X^{3}}{T A_{1} A_{2} \varphi(q)}+L^{4} \log (2 X)^{2} E(q, \boldsymbol{a}) .
$$

Proving this requires a technical result similar to [Le Boudec 2012b, Lemma 4].

Lemma 4. Let $0<v \leq 1$ and $M_{0} \in \mathbb{R}_{>0}$. Let $Y \in \mathbb{R}_{>0}$ and $Y^{\prime} \in \mathbb{R}$ be such that $0<Y-Y^{\prime} \ll v M_{0}^{2}$. Let also $A \in \mathbb{R}$ and set $M=\max \left(|A|, Y^{1 / 2}\right)$. Let $\mathscr{R} \subset \mathbb{R}$ be the set of real numbers y satisfying

$$
Y^{\prime}<\left|y^{2}+2 A y\right| \leq Y .
$$

If $M_{0} \gg M$ then we have the bound

$$
\#(\mathscr{R} \cap \mathbb{Z}) \ll v^{1 / 2} \frac{M_{0}^{2}}{M}+1 .
$$

Proof. Without using the assumption $M_{0} \gg M$, the proof of [Le Boudec 2012b, Lemma 4] shows that we have

$$
\#(\Re \cap \mathbb{Z}) \ll v \frac{M_{0}^{2}}{M}+v^{1 / 2} M_{0}+1 .
$$

Therefore, under the assumption $M_{0} \gg M$, we clearly have the claimed upper bound.

Proof of Lemma 3. If $\mathscr{Y} \cap \mathbb{Z}_{\neq 0}^{2}=\varnothing$ then the result is obvious. We therefore assume from now on that $\mathscr{S} \cap \mathbb{Z}_{\neq 0}^{2} \neq \varnothing$. We let $0<\delta, \delta^{\prime} \leq 1$ be two parameters to be selected in due course, and we set $\zeta=1+\delta$ and $\zeta^{\prime}=1+\delta^{\prime}$. In addition, we let $U$ and $V$ be variables running over the sets $\left\{ \pm \zeta^{n} \mid n \in \mathbb{Z}_{\geq-1}\right\}$ and $\left\{ \pm \zeta^{\prime n} \mid n \in \mathbb{Z}_{\geq-1}\right\}$, respectively. We define $\mathscr{I}=] U, \zeta U]$ if $U>0$ and $\mathscr{I}=[\zeta U, U$ [ if $U<0$, and define the interval $\mathscr{g}$ the same way using the variable $V$ and the parameter $\zeta^{\prime}$. We have

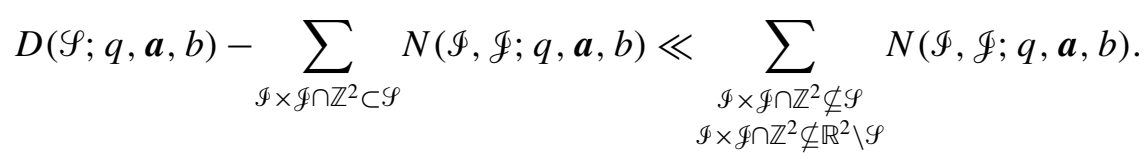


We define the quantity

$$
D(\mathscr{S} ; q)=\sum_{\mathscr{I} \times \mathscr{q} \cap \mathbb{Z}^{2} \subset \mathscr{S}} N^{*}(\mathscr{g}, \mathscr{g} ; q) .
$$

We note here that since $N^{*}(\mathscr{I}, \mathscr{q} ; q)$ is independent of $a_{1}, a_{2}$ and $b, D(\mathscr{S} ; q)$ is also independent of $a_{1}, a_{2}$ and $b$. Moreover, we have

$$
\sum_{\mathscr{I} \times \mathscr{F} \cap \mathbb{Z}^{2} \subset \mathscr{S}} N(\mathscr{I}, \mathscr{g} ; q, \boldsymbol{a}, b)-D(\mathscr{S} ; q) \ll \frac{\log (2 X)^{2}}{\delta \delta^{\prime}} E(q, \boldsymbol{a}),
$$

where we have used Lemma 2 and noted that the number of rectangles $\mathscr{I} \times \mathscr{\Phi}$ such that $\mathscr{I} \times \mathscr{g} \cap \mathbb{Z}^{2} \subset \mathscr{Y}$ is at most

$$
4\left(1+\frac{\log X}{\log \zeta}\right)\left(1+\frac{\log X}{\log \zeta^{\prime}}\right) \ll \frac{\log (2 X)^{2}}{\delta \delta^{\prime}},
$$

since $\delta, \delta^{\prime} \leq 1$. We have proved that

$$
D(\mathscr{S} ; q, \boldsymbol{a}, b)-D(\mathscr{S} ; q) \ll \sum_{\substack{\Phi \times \mathscr{G} \cap \mathbb{Z}^{2} \nsubseteq \mathscr{S} \\ \mathscr{I} \times \mathscr{G} \cap \mathbb{Z}^{2} \nsubseteq \mathbb{R}^{2} \backslash \mathscr{S}}} N(\mathscr{I}, \mathscr{g} ; q, \boldsymbol{a}, b)+\frac{\log (2 X)^{2}}{\delta \delta^{\prime}} E(q, \boldsymbol{a}) .
$$

Using the bound (2-7) for $N(\mathscr{g}, \mathscr{q} ; q, \boldsymbol{a}, b)$, we conclude that

$$
D(\mathscr{S} ; q, \boldsymbol{a}, b)-D(\mathscr{S} ; q) \ll \frac{1}{\varphi(q)} \sum_{\substack{\mathscr{S} \times \mathscr{q} \cap \mathbb{Z}^{2} \nsubseteq \mathscr{\Phi} \\ \mathscr{g} \times \mathscr{q} \cap \mathbb{Z}^{2} \nsubseteq \mathbb{R}^{2} \backslash \mathscr{S}}} \#\left(\mathscr{I} \times \mathscr{\mathscr { S }} \cap \mathbb{Z}^{2}\right)+\frac{\log (2 X)^{2}}{\delta \delta^{\prime}} E(q, \boldsymbol{a}),
$$

since the number of rectangles $\mathscr{I} \times \mathscr{g}$ satisfying $\mathscr{I} \times \mathscr{F} \cap \mathbb{Z}^{2} \nsubseteq \mathscr{S}$ and $\mathscr{I} \times \mathscr{G} \cap \mathbb{Z}^{2} \nsubseteq \mathbb{R}^{2} \backslash \mathscr{T}$ is also $\ll \log (2 X)^{2} \delta^{-1} \delta^{\prime-1}$. The sum of the right-hand side is over all the rectangles $\mathscr{I} \times \mathscr{g}$ for which $\left(\zeta^{s_{1}} U, \zeta^{\prime s_{2}} V\right) \in \mathscr{P} \cap \mathbb{Z}^{2}$ and $\left(\zeta^{t_{1}} U, \zeta^{\prime t_{2}} V\right) \in \mathbb{Z}^{2} \backslash \mathscr{Y}$ for some $\left(s_{1}, s_{2}\right) \in$ ] $0,1]^{2}$ and $\left.\left.\left(t_{1}, t_{2}\right) \in\right] 0,1\right]^{2}$. This means that one of the inequalities defining $\mathscr{Y}$ is not satisfied by $\left(\zeta^{t_{1}} U, \zeta^{\prime t_{2}} V\right)$, and we need to estimate the contribution coming from each of the conditions (2-8)-(2-11). Note that we always have the conditions

$$
\begin{aligned}
& A_{1}|U| \leq X, \\
& A_{2}|V| \leq X .
\end{aligned}
$$

In what follows, we could sometimes write strict inequalities instead of nonstrict ones, but this would not change anything in our reasoning. Let us first deal with condition (2-8). For the rectangles $\mathscr{I} \times \mathscr{F}$ described above, for some $\left.\left.\left(s_{1}, s_{2}\right) \in\right] 0,1\right]^{2}$ and $\left.\left.\left(t_{1}, t_{2}\right) \in\right] 0,1\right]^{2}$ we have 


$$
\begin{gathered}
\zeta^{s_{1}} \zeta^{\prime s_{2}} A_{1}|U| A_{2}|V|\left|\zeta^{s_{1}} A_{1} U+\zeta^{\prime s_{2}} A_{2} V-T\right| \leq T^{2} X, \\
\zeta^{t_{1}} \zeta^{\prime t_{2}} A_{1}|U| A_{2}|V|\left|\zeta^{t_{1}} A_{1} U+\zeta^{\prime t_{2}} A_{2} V-T\right|>T^{2} X .
\end{gathered}
$$

These two conditions imply respectively

$$
\left|A_{1} U+A_{2} V-T\right| \leq \frac{T^{2} X}{A_{1}|U| A_{2}|V|}+\delta A_{1}|U|+\delta^{\prime} A_{2}|V|,
$$

and

$$
\left|A_{1} U+A_{2} V-T\right|>\zeta^{-1} \zeta^{\prime-1} \frac{T^{2} X}{A_{1}|U| A_{2}|V|}-\delta A_{1}|U|-\delta^{\prime} A_{2}|V| .
$$

Setting $\Delta=\delta+\delta^{\prime}$, we thus get

$$
\zeta^{-1} \zeta^{\prime-1} \frac{T^{2} X}{A_{1}|U| A_{2}|V|}-\Delta X<\left|A_{1} U+A_{2} V-T\right| \leq \frac{T^{2} X}{A_{1}|U| A_{2}|V|}+\Delta X .
$$

Going back to the variables $u$ and $v$, it is immediate to check that

$$
|| A_{1} u+A_{2} v-T|-| A_{1} U+A_{2} V-T|| \leq \delta A_{1}|U|+\delta^{\prime} A_{2}|V| \leq \Delta X .
$$

Therefore, the inequality (2-16) gives

$$
\zeta^{-1} \zeta^{\prime-1} \frac{T^{2} X}{A_{1}|u| A_{2}|v|}-2 \Delta X<\left|A_{1} u+A_{2} v-T\right| \leq \zeta \zeta^{\prime} \frac{T^{2} X}{A_{1}|u| A_{2}|v|}+2 \Delta X .
$$

Finally, we obtain the condition

$$
\zeta^{-1} \zeta^{\prime-1} \frac{T^{2} X}{A_{1}^{2} A_{2}|v|}-4 \Delta \frac{X^{2}}{A_{1}^{2}}<|u|\left|u+\frac{A_{2}}{A_{1}} v-\frac{T}{A_{1}}\right| \leq \zeta \zeta^{\prime} \frac{T^{2} X}{A_{1}^{2} A_{2}|v|}+4 \Delta \frac{X^{2}}{A_{1}^{2}} .
$$

Since $T \leq 3 X$, we can apply Lemma 4 with

$$
M_{0}=\frac{X^{3 / 2}}{A_{1} A_{2}^{1 / 2}|v|^{1 / 2}}
$$

and $v=\Delta$. We see that the error we want to estimate is bounded by

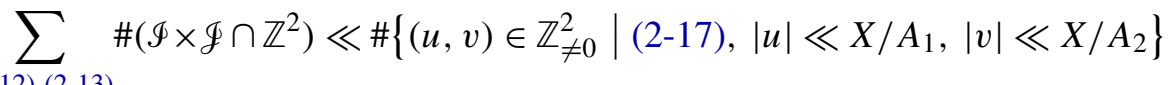

(2-16)

$$
\ll \sum_{|v| \ll X / A_{2}}\left(\Delta^{1 / 2} \frac{X^{5 / 2}}{T A_{1} A_{2}^{1 / 2}|v|^{1 / 2}}+1\right) \ll \Delta^{1 / 2} \frac{X^{3}}{T A_{1} A_{2}}+\frac{X}{A_{2}} .
$$

Using the symmetry between the variables $u$ and $v$, we see that we also have

$$
\sum_{\substack{(2-12),(2-13) \\(2-16)}} \#\left(\Phi \times \mathscr{g} \cap \mathbb{Z}^{2}\right) \ll \Delta^{1 / 2} \frac{X^{3}}{T A_{1} A_{2}}+\frac{X}{A_{1}}
$$


and thus

$$
\sum_{\substack{(2-12),(2-13) \\(2-16)}} \#\left(\mathscr{I} \times \mathscr{g} \cap \mathbb{Z}^{2}\right) \ll \Delta^{1 / 2} \frac{X^{3}}{T A_{1} A_{2}}+\frac{X}{A_{1}^{1 / 2} A_{2}^{1 / 2}}
$$

We now reason in a similar way to treat the cases of the other conditions. Let us estimate the contribution coming from condition (2-9). We see that the condition which plays the role of (2-16) in the previous case is here

$$
X-\Delta X<\left|A_{1} U+A_{2} V-T\right| \leq X+\Delta X .
$$

Furthermore, going back to the variables $u$ and $v$, we obtain

$$
X-2 \Delta X<\left|A_{1} u+A_{2} v-T\right| \leq X+2 \Delta X .
$$

We therefore find that the error in this case is bounded by

$$
\sum \#\left(\mathscr{I} \times \mathscr{g} \cap \mathbb{Z}^{2}\right) \ll \#\left\{(u, v) \in \mathbb{Z}_{\neq 0}^{2}|(2-19),| u\left|\ll X / A_{1},\right| v \mid \ll X / A_{2}\right\}
$$

$(2-18)$

$$
\ll \sum_{|v| \ll X / A_{2}}\left(\Delta \frac{X}{A_{1}}+1\right) \ll \Delta \frac{X^{2}}{A_{1} A_{2}}+\frac{X}{A_{2}} .
$$

Once again using the symmetry between the variables $u$ and $v$, we obtain

$$
\sum_{\substack{(2-12),(2-13) \\(2-18)}} \#\left(\Phi \times \mathscr{g} \cap \mathbb{Z}^{2}\right) \ll \Delta \frac{X^{2}}{A_{1} A_{2}}+\frac{X}{A_{1}^{1 / 2} A_{2}^{1 / 2}} .
$$

Finally, if $X / A_{1}<2$ then it is clear that we do not have to consider the case of condition (2-10), and if $X / A_{1} \geq 2$ then we are going to choose $\delta$ such that $X / A_{1}$ is an integer power of $\zeta$ and, as a result, we do not have to consider the case of this condition, here either. The same reasoning holds for the choice of the parameter $\delta^{\prime}$ depending on the size of the quantity $X / A_{2}$. As a consequence, we have obtained $D(\mathscr{S} ; q, \boldsymbol{a}, b)-D(\mathscr{Y} ; q) \ll \Delta^{1 / 2} \frac{X^{3}}{T A_{1} A_{2} \varphi(q)}+\frac{\log (2 X)^{2}}{\delta \delta^{\prime}} E(q, \boldsymbol{a})+\frac{X}{A_{1}^{1 / 2} A_{2}^{1 / 2} \varphi(q)}$.

Note that if $q=1$ then the result of Lemma 3 is clear since $D(\mathscr{S} ; 1, \boldsymbol{a}, b)=D^{*}(\mathscr{S} ; 1)$ and if $q>1$ then the third term of the right-hand side is dominated by one of the other two. We can always choose $\delta$ and $\delta^{\prime}$ such that $\zeta$ and $\zeta^{\prime}$ are integer powers of $X / A_{1}$ and $X / A_{2}$ respectively if these quantities are greater than or equal to 2; and we can require that, given $L \geq 1$,

$$
\delta, \delta^{\prime} \asymp \frac{1}{L^{2}} .
$$


These choices of $\delta$ and $\delta^{\prime}$ give

$$
D(\mathscr{S} ; q, \boldsymbol{a}, b)-D(\mathscr{Y} ; q) \ll \frac{1}{L} \frac{X^{3}}{T A_{1} A_{2} \varphi(q)}+L^{4} \log (2 X)^{2} E(q, \boldsymbol{a}) .
$$

Since $D(\mathscr{Y} ; q)$ does not depend on $a_{2}$ and $D^{*}(\mathscr{Y} ; q)$ is the average of $D(\mathscr{Y} ; q, \boldsymbol{a}, b)$ over $a_{2}$ coprime to $q$, averaging the last estimate over $a_{2}$ coprime to $q$ yields

$$
D^{*}(\mathscr{Y} ; q)-D(\mathscr{S} ; q) \ll \frac{1}{L} \frac{X^{3}}{T A_{1} A_{2} \varphi(q)}+L^{4} \log (2 X)^{2} E_{1}(q) .
$$

Putting these two estimates together completes the proof.

Our next aim is to approximate the cardinality which appears in $D^{*}(\mathscr{Y} ; q)$ by its corresponding two-dimensional volume. For this, we define the real-valued function

$$
h:(x, y, t) \mapsto \max \left\{|x y||x+y-t|, t^{2}|x|, t^{2}|y|, t^{2}|x+y-t|\right\} .
$$

It is immediate to check that

$$
\mathscr{Y}=\left\{(x, y) \in \mathbb{R}^{2} \mid h\left(\frac{A_{1} x}{X^{1 / 3} T^{2 / 3}}, \frac{A_{2} y}{X^{1 / 3} T^{2 / 3}}, \frac{T^{1 / 3}}{X^{1 / 3}}\right) \leq 1\right\} .
$$

We also introduce the real-valued functions

$$
g_{1}:(y, t) \mapsto \int_{h(x, y, t) \leq 1} \mathrm{~d} x, \quad g_{2}: t \mapsto \int g_{1}(y, t) \mathrm{d} y .
$$

Lemma 5. For $(y, t) \in \mathbb{R} \times \mathbb{R}_{>0}$, we have the bounds

$$
g_{1}(y, t) \ll t^{-2} \text { and } g_{2}(t) \ll 1 .
$$

Proof. The bound for $g_{1}$ is clear since $t^{2}|x| \leq 1$. To prove the bound for $g_{2}$, we use the elementary result [Derenthal 2009, Lemma 5.1]. We obtain

$$
\int_{|x y||x+y-t| \leq 1} \mathrm{~d} x \ll \min \left\{\frac{1}{|y|^{1 / 2}}, \frac{1}{|y||y-t|}\right\} .
$$

Therefore, we have

$$
g_{2}(t) \ll \int_{|y| \leq 1} \frac{\mathrm{d} y}{|y|^{1 / 2}}+\int_{|y|,|y-t| \geq 1} \frac{\mathrm{d} y}{|y||y-t|}+\int_{|y| \geq 1,|y-t| \leq 1} \frac{\mathrm{d} y}{|y|^{3 / 4}|y-t|^{1 / 2}} .
$$

The three terms of the right-hand side are easily seen to be bounded by an absolute constant, which completes the proof.

We now prove that the following result holds: 
Lemma 6. We have the estimate

$$
D^{*}(\mathscr{S} ; q)-\frac{\varphi(q)}{q^{2}} \frac{X^{2 / 3} T^{4 / 3}}{A_{1} A_{2}} g_{2}\left(\frac{T^{1 / 3}}{X^{1 / 3}}\right) \ll \frac{X^{2}}{A_{1} A_{2} q}\left(\frac{A_{1}^{1 / 2}}{X^{1 / 2}}+\frac{A_{2}^{1 / 2}}{X^{1 / 2}}\right) E_{2}(q),
$$

where

$$
E_{2}(q)=\frac{q}{\varphi(q)} \sigma_{-1 / 2}(q) \sigma_{-1}(q)
$$

Proof. We start by removing the two coprimality conditions $\operatorname{gcd}(u, q)=1$ and $\operatorname{gcd}(v, q)=1$ using Möbius inversions. We get

where

$$
D^{*}(\mathscr{S} ; q)=\frac{1}{\varphi(q)} \sum_{\ell_{1} \mid q} \mu\left(\ell_{1}\right) \sum_{\ell_{2} \mid q} \mu\left(\ell_{2}\right) C\left(\ell_{1}, \ell_{2}, \mathscr{S}\right)
$$

$$
C\left(\ell_{1}, \ell_{2}, \mathscr{S}\right)=\#\left\{\left(u^{\prime}, v^{\prime}\right) \in \mathbb{Z}_{\neq 0}^{2} \mid\left(\ell_{1} u^{\prime}, \ell_{2} v^{\prime}\right) \in \mathscr{P}\right\} .
$$

To count the number of $u^{\prime}$ to be considered, we use the estimate

$$
\#\left\{n \in \mathbb{Z}_{\neq 0} \cap\left[t_{1}, t_{2}\right]\right\}=t_{2}-t_{1}+O\left(\max \left(\left|t_{1}\right|,\left|t_{2}\right|\right)^{1 / 2}\right),
$$

which is valid for any $t_{1}, t_{2} \in \mathbb{R}$ such that $t_{1} \leq t_{2}$. We obtain

$$
\begin{aligned}
C\left(\ell_{1}, \ell_{2}, \mathscr{S}\right) & =\sum_{\substack{v^{\prime} \in \mathbb{Z} \neq 0 \\
A_{2} \ell_{2}\left|v^{\prime}\right| \leq X}}\left(\frac{X^{1 / 3} T^{2 / 3}}{A_{1} \ell_{1}} g_{1}\left(\frac{A_{2} \ell_{2} v^{\prime}}{X^{1 / 3} T^{2 / 3}}, \frac{T^{1 / 3}}{X^{1 / 3}}\right)+O\left(\frac{X^{1 / 2}}{A_{1}^{1 / 2} \ell_{1}^{1 / 2}}\right)\right) \\
& =\frac{X^{1 / 3} T^{2 / 3}}{A_{1} \ell_{1}} \sum_{\substack{v^{\prime} \in \mathbb{Z}_{\neq 0} \\
A_{2} \ell_{2}\left|v^{\prime}\right| \leq X}} g_{1}\left(\frac{A_{2} \ell_{2} v^{\prime}}{X^{1 / 3} T^{2 / 3}}, \frac{T^{1 / 3}}{X^{1 / 3}}\right)+O\left(\frac{X^{3 / 2}}{A_{1}^{1 / 2} \ell_{1}^{1 / 2} A_{2} \ell_{2}}\right) .
\end{aligned}
$$

The first bound of Lemma 5 implies that

$$
\sup _{|y| \leq X^{2 / 3} / T^{2 / 3}} g_{1}\left(y, \frac{T^{1 / 3}}{X^{1 / 3}}\right) \ll \frac{X^{2 / 3}}{T^{2 / 3}} .
$$

Since $g_{1}$ is easily seen to have a piecewise continuous derivative, this bound, an application of partial summation and a further use of the estimate (2-23) yield

$$
\sum_{\substack{v^{\prime} \in \mathbb{Z}_{\neq 0} \\ A_{2} \ell_{2}\left|v^{\prime}\right| \leq X}} g_{1}\left(\frac{A_{2} \ell_{2} v^{\prime}}{X^{1 / 3} T^{2 / 3}}, \frac{T^{1 / 3}}{X^{1 / 3}}\right)=\frac{X^{1 / 3} T^{2 / 3}}{A_{2} \ell_{2}} g_{2}\left(\frac{T^{1 / 3}}{X^{1 / 3}}\right)+O\left(\frac{X^{7 / 6}}{T^{2 / 3} A_{2}^{1 / 2} \ell_{2}^{1 / 2}}\right) .
$$

We have finally proved that

$$
C\left(\ell_{1}, \ell_{2}, \mathscr{S}\right)=\frac{1}{\ell_{1} \ell_{2}} \frac{X^{2 / 3} T^{4 / 3}}{A_{1} A_{2}} g_{2}\left(\frac{T^{1 / 3}}{X^{1 / 3}}\right)+O\left(\frac{X^{3 / 2}}{A_{1} \ell_{1} A_{2}^{1 / 2} \ell_{2}^{1 / 2}}+\frac{X^{3 / 2}}{A_{1}^{1 / 2} \ell_{1}^{1 / 2} A_{2} \ell_{2}}\right) .
$$


Putting together this estimate and the equality (2-22) completes the proof.

One of the immediate consequences of Lemmas 3 and 6 is the following result, which corresponds exactly to the setting of the proof of Theorem 1:

Lemma 7. Let $L \geq 1$ and $\mathscr{L} \geq 1$. If

$$
\frac{X}{\mathscr{L}} \leq T
$$

then we have the estimate

$$
D(\mathscr{Y} ; q, \boldsymbol{a}, b)-\frac{\varphi(q)}{q^{2}} \frac{X^{2 / 3} T^{4 / 3}}{A_{1} A_{2}} g_{2}\left(\frac{T^{1 / 3}}{X^{1 / 3}}\right) \ll E,
$$

where $E=E\left(X, T, A_{1}, A_{2}, L, \mathscr{L}, q, \boldsymbol{a}\right)$ is given by

$$
E=L^{4} \log (2 X)^{2} E(q, \boldsymbol{a})+\frac{X^{2 / 3} T^{4 / 3}}{A_{1} A_{2} q} \mathscr{L}^{4 / 3}\left(\frac{\mathscr{L}}{L}+\frac{A_{1}^{1 / 2}}{X^{1 / 2}}+\frac{A_{2}^{1 / 2}}{X^{1 / 2}}\right) E_{2}(q) .
$$

2.2. The error term. We now turn to the investigation of the error term $E\left(q, \boldsymbol{a}^{\prime}\right)$ in the particular case where $\boldsymbol{a}^{\prime}=\left(b_{1} c_{1}^{2}, b_{2} c_{2}^{2}\right)$ for $b_{1}, b_{2}, c_{1}, c_{2} \in \mathbb{Z}_{\geq 1}$. Recall that we have $\operatorname{gcd}\left(b_{1} b_{2} c_{1} c_{2}, q\right)=1$. We aim to give an upper bound for the sums of $E\left(q, \boldsymbol{a}^{\prime}\right)$ over $c_{1}$ and $c_{2}$ in some dyadic ranges. For this, we make use of the following result, which comes from the geometry of numbers and is due to Heath-Brown (see [1984, Lemma 3]). Note that this result had already been used by Browning [2006] to prove that $N_{U, H}(B)$ has the expected order of magnitude.

Lemma 8. Let $\left(v_{1}, v_{2}, v_{3}\right) \in \mathbb{Z}^{3}$ be a primitive vector, and let $W_{1}, W_{2}, W_{3} \geq 1$. The number of primitive vectors $\left(w_{1}, w_{2}, w_{3}\right) \in \mathbb{Z}^{3}$ satisfying the conditions $\left|w_{i}\right| \leq W_{i}$ for $i=1,2,3$ and the equation

$$
v_{1} w_{1}+v_{2} w_{2}+v_{3} w_{3}=0
$$

is at most

$$
12 \pi \frac{W_{1} W_{2} W_{3}}{\max \left\{\left|v_{i}\right| W_{i}\right\}}+4
$$

where the maximum is taken over $i=1,2,3$.

From now on, we let $\tau$ be the usual divisor function. Recall the definitions of $E\left(q, \boldsymbol{a}^{\prime}\right)$ and $E_{1}(q)$ given in Lemma 2. We now prove the following lemma:

Lemma 9. Let $C_{1}, C_{2} \geq \frac{1}{2}$. We have the bound

$$
\sum_{\substack{C_{i}<c_{i} \leq 2 C_{i} \\ \operatorname{gcd}\left(c_{1}, c_{2}\right)=1}}^{*} E\left(q, \boldsymbol{a}^{\prime}\right) \ll\left(C_{1} C_{2} \tau(q)+q\right) 2^{\omega(q)} E_{1}(q),
$$

where the notation $\sum^{*}$ means that the summation is restricted to integers which are coprime to $q$ and where $i$ implicitly runs over the set $\{1,2\}$. 
Proof. We have

$$
\sum_{\substack{C_{i}<c_{i} \leq 2 C_{i} \\ \operatorname{gcd}\left(c_{1}, c_{2}\right)=1}}^{*} E\left(q, \boldsymbol{a}^{\prime}\right) \ll \sum_{\substack{C_{i}<c_{i} \leq 2 C_{i} \\ \operatorname{gcd}\left(c_{1}, c_{2}\right)=1}}^{*} E_{0}\left(q, \boldsymbol{a}^{\prime}\right)+C_{1} C_{2} E_{1}(q) .
$$

The first term of the right-hand side is at most

$$
\sum_{d \mid q} d \sum_{0<|r|,|s| \leq q / 2}|r|^{-1}|s|^{-1} \sum_{\substack{C_{i}<c_{i} \leq 2 C_{i} \\ \operatorname{gcd}\left(c_{1}, c_{2}\right)=1 \\ b_{1} c_{1}^{2} s-b_{2} c_{2}^{2} r \equiv 0(\bmod d)}}^{*} 1 .
$$

Let us set $g=\operatorname{gcd}(r, s, d)$ and $s^{\prime}=s / g, r^{\prime}=r / g$ and $d^{\prime}=d / g$. We have

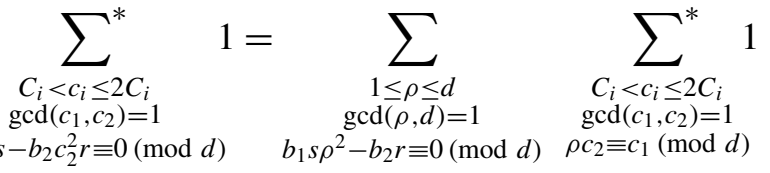

$$
\begin{aligned}
& =\sum_{\substack{1 \leq \rho \leq d \\
\operatorname{gcd}(\rho, d)=1 \\
b_{1} s^{\prime} \rho^{2}-b_{2} r^{\prime} \equiv 0\left(\bmod d^{\prime}\right)}} \sum_{\begin{array}{c}
C_{i}<c_{i} \leq 2 C_{i} \\
\operatorname{gcd}\left(c_{1}, c_{2}\right)=1 \\
\rho c_{2} \equiv c_{1}(\bmod d)
\end{array}}^{*} 1 \\
& =\sum_{\substack{1 \leq \rho \leq d \\
\operatorname{gcd}(\rho, d)=1 \\
\rho^{2}-\left(b_{1} s^{\prime}\right)^{-1} b_{2} r^{\prime} \equiv 0\left(\bmod d^{\prime}\right)}} \sum_{\begin{array}{c}
C_{i}<c_{i} \leq 2 C_{i} \\
\operatorname{gcd}\left(c_{1}, c_{2}\right)=1 \\
\rho c_{2} \equiv c_{1}(\bmod d)
\end{array}}^{*} 1,
\end{aligned}
$$

since $\operatorname{gcd}\left(b_{1} b_{2}, d^{\prime}\right)=1$ and $\operatorname{gcd}\left(r^{\prime}, s^{\prime}, d^{\prime}\right)=1$, and where $\left(b_{1} s^{\prime}\right)^{-1}$ denotes the inverse of $b_{1} s^{\prime}$ modulo $d^{\prime}$. Using Lemma 8, we get

$$
\sum_{\substack{C_{i}<c_{i} \leq 2 C_{i} \\ \operatorname{gcd}\left(c_{1}, c_{2}\right)=1 \\ \rho c_{2} \equiv c_{1}(\bmod d)}}^{*} 1 \ll \frac{C_{1} C_{2}}{d}+1
$$

As a consequence, we have proved that

$$
\sum_{\substack{C_{i}<c_{i} \leq 2 C_{i} \\ \operatorname{gcd}\left(c_{1}, c_{2}\right)=1 \\ s-b_{2} c_{2}^{2} r \equiv 0(\bmod d)}}^{*} 1 \ll \operatorname{gcd}(r, s, d) 2^{\omega(d)}\left(\frac{C_{1} C_{2}}{d}+1\right) .
$$

Finally, we easily get 


$$
\begin{aligned}
\sum_{d \mid q} d \sum_{0<|r|,|s| \leq q / 2}|r|^{-1}|s|^{-1} \frac{\operatorname{gcd}(r, s, d) 2^{\omega(d)}}{d} & \ll \sum_{d \mid q} 2^{\omega(d)} \sum_{e \mid d} e \sum_{\substack{0<|r|,|s| \leq q / 2 \\
e|r, e| s}}|r|^{-1}|s|^{-1} \\
& \ll 2^{\omega(q)} \tau(q) \sigma_{-1}(q)(\log q)^{2} \\
& \ll 2^{\omega(q)} \tau(q) E_{1}(q),
\end{aligned}
$$

and, as in the proof of Lemma 2, we obtain

$$
\sum_{d \mid q} d \sum_{0<|r|,|s| \leq q / 2}|r|^{-1}|s|^{-1} \operatorname{gcd}(r, s, d) 2^{\omega(d)} \ll q 2^{\omega(q)} E_{1}(q) .
$$

As a result, we have proved that

$$
\sum_{\substack{C_{i}<c_{i} \leq 2 C_{i} \\ \operatorname{gcd}\left(c_{1}, c_{2}\right)=1}}^{*} E_{0}\left(q, \boldsymbol{a}^{\prime}\right) \ll\left(C_{1} C_{2} \tau(q)+q\right) 2^{\omega(q)} E_{1}(q),
$$

which completes the proof.

2.3. Arithmetic functions. We now introduce several arithmetic functions which will appear along the proof of Theorem 1 . We set

$$
\begin{aligned}
& \varphi^{*}(n)=\prod_{p \mid n}\left(1-\frac{1}{p}\right), \\
& \varphi^{\curlyvee}(n)=\prod_{p \mid n}\left(1-\frac{1}{p}\right)^{-2}\left(1+\frac{2}{p}\right)^{-1},
\end{aligned}
$$

and also, for $a, b \in \mathbb{Z}_{\geq 1}$,

$$
\psi_{a}(n)=\prod_{\substack{p \mid n \\ p \nmid a}}\left(1-\frac{1}{p}\right)^{2}\left(1-\frac{1}{p-1}\right)
$$

and

$$
\psi_{a, b}(n)= \begin{cases}\psi_{a}(n) & \text { if } \operatorname{gcd}(n, b)=1, \\ 0 & \text { otherwise }\end{cases}
$$

Following the straightforward reasoning of the proofs of [Le Boudec 2012b, Lemmas 5, 6], we easily obtain the following result:

Lemma 10. Let $0<\gamma \leq 1$ be fixed. Let $0 \leq t_{1}<t_{2}$, and set $I=\left[t_{1}, t_{2}\right]$. Let $g: \mathbb{R}_{>0} \rightarrow \mathbb{R}$ be a function with a piecewise continuous derivative on I whose sign changes at most $R_{g}(I)$ times on $I$. We have

$$
\sum_{n \in I \cap \mathbb{Z}_{>0}} \psi_{a, b}(n) g(n)=\Upsilon \Psi(a, b) \int_{I} g(t) \mathrm{d} t+O_{\gamma}\left(\sigma_{-\gamma / 2}(a b) t_{2}^{\gamma} M_{I}(g)\right),
$$


where

$$
\Upsilon=\prod_{p} \varphi^{\curlyvee}(p)^{-1}, \quad \Psi(a, b)=\varphi^{*}(b) \varphi^{\curlyvee}(a b),
$$

and

$$
M_{I}(g)=\left(1+R_{g}(I)\right) \sup _{t \in I \cap \mathbb{R}_{>0}}|g(t)|
$$

\section{The universal torsor}

In this section we define a bijection between the set of rational points of bounded height on $U$ and a certain set of integral points on the hypersurface defined in the introduction. The universal torsor corresponding to our present problem was first determined by Hassett and Tschinkel [2004] and then used by Browning [2006] to prove the lower and upper bounds of the expected order of magnitude for $N_{U, H}(B)$. We employ the notation used in [Derenthal 2014]. Let $\mathscr{T}(B)$ be the set of $\left(\eta_{1}, \ldots, \eta_{10}\right) \in \mathbb{Z}_{>0}^{7} \times \mathbb{Z}_{\neq 0}^{3}$ satisfying the equation

$$
\eta_{2} \eta_{5}^{2} \eta_{8}+\eta_{3} \eta_{6}^{2} \eta_{9}+\eta_{4} \eta_{7}^{2} \eta_{10}-\eta_{1} \eta_{2} \eta_{3} \eta_{4} \eta_{5} \eta_{6} \eta_{7}=0
$$

the coprimality conditions

$$
\begin{aligned}
\operatorname{gcd}\left(\eta_{10}, \eta_{1} \eta_{2} \eta_{3} \eta_{4} \eta_{5} \eta_{6}\right) & =1, \\
\operatorname{gcd}\left(\eta_{9}, \eta_{1} \eta_{2} \eta_{3} \eta_{4} \eta_{5} \eta_{7}\right) & =1, \\
\operatorname{gcd}\left(\eta_{8}, \eta_{1} \eta_{2} \eta_{3} \eta_{4} \eta_{6} \eta_{7}\right) & =1, \\
\operatorname{gcd}\left(\eta_{1}, \eta_{5} \eta_{6} \eta_{7}\right) & =1, \\
\operatorname{gcd}\left(\eta_{2} \eta_{5}, \eta_{3} \eta_{4} \eta_{6} \eta_{7}\right) & =1, \\
\operatorname{gcd}\left(\eta_{3} \eta_{6}, \eta_{4} \eta_{7}\right) & =1,
\end{aligned}
$$

and the height conditions

$$
\begin{aligned}
\left|\eta_{8} \eta_{9} \eta_{10}\right| & \leq B, \\
\eta_{1}^{2} \eta_{2}^{2} \eta_{3} \eta_{4} \eta_{5}^{2}\left|\eta_{8}\right| & \leq B, \\
\eta_{1}^{2} \eta_{2} \eta_{3}^{2} \eta_{4} \eta_{6}^{2}\left|\eta_{9}\right| & \leq B, \\
\eta_{1}^{2} \eta_{2} \eta_{3} \eta_{4}^{2} \eta_{7}^{2}\left|\eta_{10}\right| & \leq B .
\end{aligned}
$$

Lemma 11.

$$
N_{U, H}(B)=\# \mathscr{T}(B) .
$$

Proof. It is sufficient to show that the counting problem defined by the set $\mathscr{T}(B)$ is equivalent to the one described in [Browning 2006, Section 4], which we call $\mathscr{T}^{\prime}(B)$ and which is defined exactly as $\mathscr{T}(B)$ except that the condition (3-5) is replaced by the condition $\left|\mu\left(\eta_{2} \eta_{3} \eta_{4}\right)\right|=1$. 
For $i=2,3,4$, there is only one way to write $\eta_{i}=\eta_{i}^{\prime} \eta_{i}^{\prime \prime 2}$ in such a way that $\eta_{i}^{\prime}$ is squarefree. Setting $\eta_{i+3}^{\prime}=\eta_{i+3} \eta_{i}^{\prime \prime}$ and $\eta_{1}^{\prime}=\eta_{1} \eta_{2}^{\prime \prime} \eta_{3}^{\prime \prime} \eta_{4}^{\prime \prime}$, we claim that the translation between the two counting problems is achieved via the map

$$
S:\left(\eta_{1}, \eta_{2}, \eta_{3}, \eta_{4}, \eta_{5}, \eta_{6}, \eta_{7}\right) \mapsto\left(\eta_{1}^{\prime}, \eta_{2}^{\prime}, \eta_{3}^{\prime}, \eta_{4}^{\prime}, \eta_{5}^{\prime}, \eta_{6}^{\prime}, \eta_{7}^{\prime}\right)
$$

Indeed, (3-1) and the height conditions (3-8)-(3-11) are invariant under $S$. Also, the coprimality conditions (3-2), (3-3), (3-4), (3-6) and (3-7) are preserved under $S$, and the condition (3-5) is replaced by the condition $\left|\mu\left(\eta_{2}^{\prime} \eta_{3}^{\prime} \eta_{4}^{\prime}\right)\right|=1$, which completes the proof.

\section{Calculation of Peyre's constant}

Peyre [1995] gives an interpretation for the constant $c_{V, H}$ appearing in the main term of $N_{U, H}(B)$ in Theorem 1. In our specific case, we have

$$
c_{V, H}=\alpha(\tilde{V}) \beta(\tilde{V}) \omega_{H}(\tilde{V}),
$$

where $\widetilde{V}$ denotes the minimal desingularization of $V$. The definitions of these three quantities are omitted (the reader should refer to [Peyre 1995] or to Section 4 of [Le Boudec 2012a] for some more details in an identical setting). Using the work of Derenthal, Joyce and Teitler [Derenthal et al. 2008, Theorem 1.3], it is easy to compute the constant $\alpha(\tilde{V})$. We find

$$
\alpha(\widetilde{V})=\frac{1}{120} \cdot \frac{1}{\# W\left(\mathrm{D}_{4}\right)}=\frac{1}{23040},
$$

where $W\left(\mathrm{D}_{4}\right)$ stands for the Weyl group associated to the Dynkin diagram of the singularity $\mathrm{D}_{4}$. Here, we have used $\# W\left(\mathrm{D}_{n}\right)=2^{n-1} n$ ! for any $n \geq 4$. In addition, $\beta(\widetilde{V})=1$ since $V$ is split over $\mathbb{Q}$. Finally, $\omega_{H}(\tilde{V})$ is given by

$$
\omega_{H}(\widetilde{V})=\omega_{\infty} \prod_{p}\left(1-\frac{1}{p}\right)^{7} \omega_{p}
$$

where $\omega_{\infty}$ and $\omega_{p}$ are the archimedean and $p$-adic densities respectively. Loughran [2010, Lemma 2.3] has shown that we have

$$
\omega_{p}=1+\frac{7}{p}+\frac{1}{p^{2}}
$$

Let us calculate $\omega_{\infty}$. Let $\boldsymbol{x}=\left(x_{0}, x_{1}, x_{2}, x_{3}\right)$ and $f(\boldsymbol{x})=x_{0}\left(x_{1}+x_{2}+x_{3}\right)^{2}-x_{1} x_{2} x_{3}$. We parametrize the points of $V$ with $x_{1}, x_{2}$ and $x_{3}$. We have

$$
\frac{\partial f}{\partial x_{0}}(\boldsymbol{x})=\left(x_{1}+x_{2}+x_{3}\right)^{2},
$$


and since $\boldsymbol{x}=-\boldsymbol{x} \in \mathbb{P}^{3}$, we obtain

$$
\omega_{\infty}=\frac{1}{2} \iiint_{\left|x_{1} x_{2} x_{3}\right| /\left(x_{1}+x_{2}+x_{3}\right)^{2},\left|x_{1}\right|,\left|x_{2}\right|,\left|x_{3}\right| \leq 1} \frac{\mathrm{d} x_{1} \mathrm{~d} x_{2} \mathrm{~d} x_{3}}{\left(x_{1}+x_{2}+x_{3}\right)^{2}} .
$$

Recall the definition (2-20) of the function $h$. The change of variables defined by $x_{1}=t^{2} x, x_{2}=t^{2} y$ and $x_{3}=-t^{2}(x+y-t)$ yields

$$
\omega_{\infty}=\frac{3}{2} \iiint_{h(x, y, t) \leq 1} \mathrm{~d} x \mathrm{~d} y \mathrm{~d} t=3 \iiint_{t>0, h(x, y, t) \leq 1} \mathrm{~d} x \mathrm{~d} y \mathrm{~d} t .
$$

\section{Proof of the main theorem}

5.1. Restriction of the domain. Note that in the torsor equation (3-1) the first three terms are at most $B / \eta_{1}^{2} \eta_{2} \eta_{3} \eta_{4}$ (by the height conditions (3-9)-(3-11)), and thus we have

$$
\eta_{1}^{3} \eta_{2}^{2} \eta_{3}^{2} \eta_{4}^{2} \eta_{5} \eta_{6} \eta_{7} \leq 3 B
$$

From now on, for $n \in \mathbb{Z}_{\geq 1}$ we denote by $\operatorname{sq}(n)$ the unique positive integer such that $\operatorname{sq}(n)^{2} \mid n$ and $n / \operatorname{sq}(n)^{2}$ is squarefree. Note that for two coprime integers $m, n \in \mathbb{Z}_{\geq 1}$, we have $\mathrm{sq}(m n)=\operatorname{sq}(m) \operatorname{sq}(n)$.

We now need to show that we can assume along the proof that

$$
\eta_{1} \operatorname{sq}\left(\eta_{2} \eta_{3} \eta_{4}\right) \geq B^{15 / \log \log B},
$$

and, in addition, that

$$
\eta_{1}^{3} \eta_{2}^{2} \eta_{3}^{2} \eta_{4}^{2} \eta_{5} \eta_{6} \eta_{7} \geq \frac{B}{\log \log B}
$$

The proof of Lemma 11 shows that we can make use of the estimates in [Browning 2006, Section 6] to prove that the contributions to $N_{U, H}(B)$ coming from those $\left(\eta_{1}, \ldots, \eta_{10}\right) \in \mathscr{T}(B)$ which do not satisfy one of the two inequalities (5-1) and (5-2) are actually negligible.

We start by proving a lemma:

Lemma 12. Let $M(B)$ be the overall contribution to $N_{U, H}(B)$ coming from those $\left(\eta_{1}, \ldots, \eta_{10}\right) \in \mathscr{T}(B)$ such that $\eta_{1} \operatorname{sq}\left(\eta_{2} \eta_{3} \eta_{4}\right) \leq B^{15 / \log \log B}$. We have

$$
M(B) \ll \frac{B(\log B)^{6}}{\log \log B} .
$$

Proof. Recall the notation introduced in the proof of Lemma 11. We note that the condition $\eta_{1} \operatorname{sq}\left(\eta_{2} \eta_{3} \eta_{4}\right) \leq B^{15 / \log \log B}$ is equivalent to $\eta_{1}^{\prime} \leq B^{15 / \log \log B}$.

For $i=1, \ldots, 10$ we let $Y_{i}$ be variables running over the set $\left\{2^{n} \mid n \geq-1\right\}$. By counting the number of $\left(\eta_{1}^{\prime}, \ldots, \eta_{10}^{\prime}\right) \in \mathscr{T}^{\prime}(B)$ which satisfy $Y_{i}<\left|\eta_{i}^{\prime}\right| \leq 2 Y_{i}$ for $i=1, \ldots, 10$, we claim that [Browning 2006, Sections 6.1, 6.2] gives 


$$
\begin{aligned}
M(B) \ll B & (\log B)^{5}+\sum_{Y_{i}} X_{0}^{1 / 2} X_{1}^{1 / 6} X_{2}^{1 / 6} X_{3}^{1 / 6} \\
& +\sum_{Y_{i}} \max _{\{i, j, k\}=\{2,3,4\}}\left\{\frac{Y_{1} Y_{2} Y_{3} Y_{4} Y_{5} Y_{6} Y_{7} Y_{8} Y_{9} Y_{10}}{Y_{k+6} \max \left\{Y_{i} Y_{i+3}^{2} Y_{i+6}, Y_{j} Y_{j+3}^{2} Y_{j+6}, Z_{k}\right\}}\right\},
\end{aligned}
$$

where the two sums are over the $Y_{i}, i=1, \ldots, 10$, subject to the inequalities

$$
\begin{aligned}
Y_{8} Y_{9} Y_{10} & \leq B, \\
Y_{1}^{2} Y_{2}^{2} Y_{3} Y_{4} Y_{5}^{2} Y_{8} & \leq B, \\
Y_{1}^{2} Y_{2} Y_{3}^{2} Y_{4} Y_{6}^{2} Y_{9} & \leq B, \\
Y_{1}^{2} Y_{2} Y_{3} Y_{4}^{2} Y_{7}^{2} Y_{10} & \leq B,
\end{aligned}
$$

and also

$$
Y_{1} \leq B^{15 / \log \log B},
$$

and where $X_{0}, X_{1}, X_{2}, X_{3}$ denote the left-hand sides of the inequalities (5-4), (5-5), (5-6) and (5-7) respectively, and finally, for $k \in\{2,3,4\}, Z_{k}$ is defined by

$$
Z_{k}= \begin{cases}Y_{k} Y_{k+3}^{2} Y_{k+6} & \text { if } Y_{k} Y_{k+3}^{2} Y_{k+6} \geq Y_{1} Y_{2} Y_{3} Y_{4} Y_{5} Y_{6} Y_{7}, \\ 1 & \text { otherwise. }\end{cases}
$$

Let us explain briefly how the upper bound (5-3) can be deduced from Browning's work without making use of the condition (5-8). It is useful to note that our variables $Y_{i}, i=1, \ldots, 10$, and $X_{j}, j=0, \ldots, 3$, correspond respectively to Browning's variables $S_{0}, U_{1}, U_{2}, U_{3}, S_{1}, S_{2}, S_{3}, Y_{1}, Y_{2}, Y_{3}$ and $X_{4}, X_{1}, X_{2}, X_{3}$. First, the second term of the right-hand side of [ibid., (6.26)] is equal to

$$
\frac{\left(X_{0} X_{1} X_{2} X_{3}\right)^{1 / 4}}{Y_{1}^{1 / 2}}\left(1+\frac{\log B}{\left(Y_{8} Y_{9} Y_{10}\right)^{1 / 16}} \max _{k \in\{2,3,4\}} Y_{k+6}^{1 / 16}\right)
$$

in our notation, and is easily seen to have overall contribution $B(\log B)^{5}$. As a result, the right side of [ibid., (6.29)] can actually be replaced by (in our notation)

$$
B(\log B)^{5}+\sum_{Y_{i}} X_{0}^{1 / 2} X_{1}^{1 / 6} X_{2}^{1 / 6} X_{3}^{1 / 6}
$$

Taking into account [ibid., (6.31)], we see that the right-hand side of the upper bound in [ibid., Proposition 4] can also be replaced by (5-9). Then, we note that the first term of the right-hand side of the upper bound in [ibid., Lemma 13] has overall contribution $B(\log B)^{4}$. This implies that the right-hand side of the upper bound in [ibid., Proposition 5] can be replaced by, in our notation,

$$
B(\log B)^{4}+\sum_{Y_{i}} \max _{\{i, j, k\}=\{2,3,4\}}\left\{\frac{Y_{1} Y_{2} Y_{3} Y_{4} Y_{5} Y_{6} Y_{7} Y_{8} Y_{9} Y_{10}}{Y_{k+6} \max \left\{Y_{i} Y_{i+3}^{2} Y_{i+6}, Y_{j} Y_{j+3}^{2} Y_{j+6}, Z_{k}\right\}}\right\} .
$$


This concludes the proof of the upper bound (5-3).

Let us denote by $\mathcal{N}_{1}(B)$ and $\mathcal{N}_{2}(B)$ the respective contributions of the two sums in (5-3). In the following estimations, the notation $\sum_{\widehat{Y}_{j}}$ indicates that the summation is over all the $Y_{i}$ with $i \neq j$. We start by investigating the quantity $\mathcal{N}_{1}(B)$ by summing over $Y_{5}, Y_{6}$ and $Y_{7}$ using, respectively, the conditions (5-5), (5-6) and (5-7). We get

$$
\begin{aligned}
\mathcal{N}_{1}(B) & =\sum_{Y_{i}} Y_{1} Y_{2}^{2 / 3} Y_{3}^{2 / 3} Y_{4}^{2 / 3} Y_{5}^{1 / 3} Y_{6}^{1 / 3} Y_{7}^{1 / 3} Y_{8}^{2 / 3} Y_{9}^{2 / 3} Y_{10}^{2 / 3} \\
& \ll B^{1 / 2} \sum_{\widehat{Y}_{5}, \widehat{Y}_{6}, \widehat{Y}_{7}} Y_{8}^{1 / 2} Y_{9}^{1 / 2} Y_{10}^{1 / 2} \ll B \sum_{\widehat{Y}_{5}, \widehat{Y}_{6}, \widehat{Y}_{7}, \widehat{Y}_{8}} 1 \ll \frac{B(\log B)^{6}}{\log \log B},
\end{aligned}
$$

where we have used the condition (5-4) to sum over $Y_{8}$ and the condition (5-8) to sum over $Y_{1}$. We now deal with the quantity $\mathcal{N}_{2}(B)$. We only treat the case where $(i, j, k)=(2,3,4)$, since the others are all identical. Note that if $Z_{4}=Y_{4} Y_{7}^{2} Y_{10}$ then $\mathcal{N}_{2}(B) \leq \mathcal{N}_{1}(B)$. Thus, we only need to deal with the case where $Z_{4}=1$. In addition, we proceed without loss of generality under the assumption that $Y_{2} Y_{5}^{2} Y_{8} \leq Y_{3} Y_{6}^{2} Y_{9}$. We first use this condition to sum over $Y_{5}$, and then we sum over $Y_{7}$ and $Y_{8}$ using the conditions (5-7) and (5-4) respectively. We get

$$
\begin{aligned}
\mathcal{N}_{2}(B) & \ll \sum_{Y_{i}} Y_{1} Y_{2} Y_{4} Y_{5} Y_{6}^{-1} Y_{7} Y_{8} \ll \sum_{\widehat{Y}_{5}} Y_{1} Y_{2}^{1 / 2} Y_{3}^{1 / 2} Y_{4} Y_{7} Y_{8}^{1 / 2} Y_{9}^{1 / 2} \\
& \ll B^{1 / 2} \sum_{\widehat{Y}_{5}, \widehat{Y}_{7}} Y_{8}^{1 / 2} Y_{9}^{1 / 2} Y_{10}^{-1 / 2} \ll B \sum_{\widehat{Y}_{5}, \widehat{Y}_{7}, \widehat{Y}_{8}} Y_{10}^{-1} \ll \frac{B(\log B)^{6}}{\log \log B},
\end{aligned}
$$

which completes the proof of Lemma 12.

The following lemma proves that the contribution to $N_{U, H}(B)$ coming from those $\left(\eta_{1}, \ldots, \eta_{10}\right) \in \mathscr{T}(B)$ which are subject to the stronger condition

$$
\eta_{1}^{3} \eta_{2}^{2} \eta_{3}^{2} \eta_{4}^{2} \eta_{5} \eta_{6} \eta_{7} \leq \frac{B}{\log \log B}
$$

is negligible.

Lemma 13. Let $\mathcal{M}^{\prime}(B)$ be the overall contribution to $N_{U, H}(B)$ coming from those $\left(\eta_{1}, \ldots, \eta_{10}\right) \in \mathscr{T}(B)$ such that

$$
\eta_{1}^{3} \eta_{2}^{2} \eta_{3}^{2} \eta_{4}^{2} \eta_{5} \eta_{6} \eta_{7} \leq \frac{B}{\log \log B}
$$

We have

$$
\mathcal{M}^{\prime}(B) \ll \frac{B(\log B)^{6}}{(\log \log B)^{1 / 6}} .
$$


Proof. We proceed as in the proof of Lemma 12, with the same notation. We have $\mathcal{M}^{\prime}(B) \ll B(\log B)^{5}+\sum_{Y_{i}} X_{0}^{1 / 2} X_{1}^{1 / 6} X_{2}^{1 / 6} X_{3}^{1 / 6}$

$$
+\sum_{Y_{i}} \max _{\{i, j, k\}=\{2,3,4\}}\left\{\frac{Y_{1} Y_{2} Y_{3} Y_{4} Y_{5} Y_{6} Y_{7} Y_{8} Y_{9} Y_{10}}{Y_{k+6} \max \left\{Y_{i} Y_{i+3}^{2} Y_{i+6}, Y_{j} Y_{j+3}^{2} Y_{j+6}, Z_{k}\right\}}\right\},
$$

where the two sums are over the dyadic variables $Y_{i}, i=1, \ldots, 10$, subject to the inequalities (5-4)-(5-7) and

$$
Y_{1}^{3} Y_{2}^{2} Y_{3}^{2} Y_{4}^{2} Y_{5} Y_{6} Y_{7} \leq \frac{B}{\log \log B} .
$$

Let us denote by $\mathcal{N}_{1}^{\prime}(B)$ and $\mathcal{N}_{2}^{\prime}(B)$ the respective contributions of the two sums in (5-10). Combining conditions (5-4) and (5-5), we get

$$
Y_{1}^{1 / 4} Y_{2}^{1 / 4} Y_{3}^{1 / 8} Y_{4}^{1 / 8} Y_{5}^{1 / 4} Y_{8} Y_{9}^{7 / 8} Y_{10}^{7 / 8} \leq B
$$

We start by bounding the contribution of the quantity $\mathcal{N}_{1}^{\prime}(B)$ by summing successively over $Y_{8}, Y_{9}$ and $Y_{10}$ using the conditions (5-12), (5-6) and (5-7) respectively. We deduce that

$$
\begin{aligned}
\mathcal{N}_{1}^{\prime}(B) & =\sum_{Y_{i}} Y_{1} Y_{2}^{2 / 3} Y_{3}^{2 / 3} Y_{4}^{2 / 3} Y_{5}^{1 / 3} Y_{6}^{1 / 3} Y_{7}^{1 / 3} Y_{8}^{2 / 3} Y_{9}^{2 / 3} Y_{10}^{2 / 3} \\
& \ll B^{2 / 3} \sum_{\widehat{Y}_{8}} Y_{1}^{5 / 6} Y_{2}^{1 / 2} Y_{3}^{7 / 12} Y_{4}^{7 / 12} Y_{5}^{1 / 6} Y_{6}^{1 / 3} Y_{7}^{1 / 3} Y_{9}^{1 / 12} Y_{10}^{1 / 12} \\
& \ll B^{5 / 6} \sum_{\widehat{Y}_{8}, \widehat{Y}_{9}, \widehat{Y}_{10}} Y_{1}^{1 / 2} Y_{2}^{1 / 3} Y_{3}^{1 / 3} Y_{4}^{1 / 3} Y_{5}^{1 / 6} Y_{6}^{1 / 6} Y_{7}^{1 / 6} \\
& \ll \frac{B}{(\log \log B)^{1 / 6}} \sum_{\widehat{Y}_{7}, \widehat{Y}_{8}, \widehat{Y}_{9}, \widehat{Y}_{10}} 1 \ll \frac{B(\log B)^{6}}{(\log \log B)^{1 / 6}},
\end{aligned}
$$

where we have summed over $Y_{7}$ using the condition (5-11). We now turn to the case of the quantity $\mathcal{N}_{2}^{\prime}(B)$. As in the proof of Lemma 12 , we only treat the case where $(i, j, k)=(2,3,4)$ and we work under the assumptions that $Z_{4}=1$ and thus

$$
Y_{4} Y_{7}^{2} Y_{10} \leq Y_{1} Y_{2} Y_{3} Y_{4} Y_{5} Y_{6} Y_{7}
$$

and $Y_{2} Y_{5}^{2} Y_{8} \leq Y_{3} Y_{6}^{2} Y_{9}$. Combining conditions (5-11) and (5-13), we get

$$
Y_{1}^{2} Y_{2} Y_{3} Y_{4}^{2} Y_{7}^{2} Y_{10} \leq \frac{B}{\log \log B}
$$


We first use the condition $Y_{2} Y_{5}^{2} Y_{8} \leq Y_{3} Y_{6}^{2} Y_{9}$ to sum over $Y_{5}$, and then we sum over $Y_{8}$ and $Y_{7}$ using the conditions (5-4) and (5-14) respectively. We deduce

$$
\begin{aligned}
\mathcal{N}_{2}^{\prime}(B) & \ll \sum_{Y_{i}} Y_{1} Y_{2} Y_{4} Y_{5} Y_{6}^{-1} Y_{7} Y_{8} \ll \sum_{\widehat{Y}_{5}} Y_{1} Y_{2}^{1 / 2} Y_{3}^{1 / 2} Y_{4} Y_{7} Y_{8}^{1 / 2} Y_{9}^{1 / 2} \\
& \ll B^{1 / 2} \sum_{\widehat{Y}_{5}, \widehat{Y}_{8}} Y_{1} Y_{2}^{1 / 2} Y_{3}^{1 / 2} Y_{4} Y_{7} Y_{10}^{-1 / 2} \\
& \ll \frac{B}{(\log \log B)^{1 / 2}} \sum_{\widehat{Y}_{5}, \widehat{Y}_{7}, \widehat{Y}_{8}} Y_{10}^{-1} \ll \frac{B(\log B)^{6}}{(\log \log B)^{1 / 2}},
\end{aligned}
$$

which completes the proof of Lemma 13.

5.2. Setting up. First, we recall that we have the following condition (given at the beginning of Section 5.1):

$$
\eta_{1}^{3} \eta_{2}^{2} \eta_{3}^{2} \eta_{4}^{2} \eta_{5} \eta_{6} \eta_{7} \leq 3 B
$$

It is easy to check that the symmetry between the three quantities $\eta_{2} \eta_{5}^{2}, \eta_{3} \eta_{6}^{2}$ and $\eta_{4} \eta_{7}^{2}$ is demonstrated by the action of $\mathfrak{S}_{3}$ on $\left\{\left(\eta_{2}, \eta_{5}, \eta_{8}\right),\left(\eta_{3}, \eta_{6}, \eta_{9}\right),\left(\eta_{4}, \eta_{7}, \eta_{10}\right)\right\}$. Throughout the proof, we will assume that

$$
\eta_{4} \eta_{7}^{2} \leq \eta_{2} \eta_{5}^{2}, \eta_{3} \eta_{6}^{2}
$$

The following lemma proves that we just need to multiply our future main term by a factor of 3 to take this new assumption into account.

Lemma 14. Let $N_{0}(B)$ be the total number of $\left(\eta_{1}, \ldots, \eta_{10}\right) \in \mathcal{T}(B)$ such that $\eta_{2} \eta_{5}^{2}=\eta_{4} \eta_{7}^{2}$ or $\eta_{3} \eta_{6}^{2}=\eta_{4} \eta_{7}^{2}$. We have the upper bound

$$
N_{0}(B) \ll B(\log B)^{3} .
$$

Proof. By symmetry, we only need to treat the case of the condition $\eta_{3} \eta_{6}^{2}=\eta_{4} \eta_{7}^{2}$. This equality and the condition $\operatorname{gcd}\left(\eta_{3} \eta_{6}, \eta_{4} \eta_{7}\right)=1$ imply that $\eta_{3}=\eta_{4}=\eta_{6}=\eta_{7}=1$. In this situation, the torsor equation is simply

$$
\eta_{2} \eta_{5}^{2} \eta_{8}+\eta_{9}+\eta_{10}-\eta_{1} \eta_{2} \eta_{5}=0
$$

Thus, $N_{0}(B)$ is bounded by the number of $\left(\eta_{1}, \eta_{2}, \eta_{5}, \eta_{8}, \eta_{9}\right) \in \mathbb{Z}_{>0}^{3} \times \mathbb{Z}_{\neq 0}^{2}$ satisfying

$$
\left|\eta_{8} \eta_{9}\right|\left|\eta_{2} \eta_{5}^{2} \eta_{8}+\eta_{9}-\eta_{1} \eta_{2} \eta_{5}\right| \leq B \quad \text { and } \quad \eta_{1}^{2} \eta_{2}^{2} \eta_{5}^{2}\left|\eta_{8}\right| \leq B
$$

Using [Le Boudec 2012a, Lemma 1] to count the number of $\eta_{9}$ satisfying the first of these two inequalities, we obtain

$$
N_{0}(B) \ll \sum_{\substack{\eta_{1}, \eta_{2}, \eta_{5}, \eta_{8} \\ \eta_{1}^{2} \eta_{2}^{2} \eta_{5}^{2}\left|\eta_{8}\right| \leq B}}\left(\frac{B^{1 / 2}}{\left|\eta_{8}\right|^{1 / 2}}+1\right) \ll B(\log B)^{3},
$$


as wished.

Let $N(B)$ be the overall contribution of those $\left(\eta_{1}, \ldots, \eta_{10}\right) \in \mathscr{T}(B)$ subject to the conditions

$$
\begin{aligned}
\eta_{4} \eta_{7}^{2} & \leq \eta_{2} \eta_{5}^{2}, \eta_{3} \eta_{6}^{2}, \\
B^{15 / \log \log B} & \leq \eta_{1} \operatorname{sq}\left(\eta_{2} \eta_{3} \eta_{4}\right), \\
\frac{B}{\log \log B} & \leq \eta_{1}^{3} \eta_{2}^{2} \eta_{3}^{2} \eta_{4}^{2} \eta_{5} \eta_{6} \eta_{7} .
\end{aligned}
$$

Lemmas 11-14 give us the following result:

Lemma 15.

$$
N_{U, H}(B)=3 N(B)+O\left(\frac{B(\log B)^{6}}{(\log \log B)^{1 / 6}}\right) .
$$

The end of the proof is devoted to the estimation of $N(B)$.

5.3. Application of Lemma 7. The idea of the proof is to view the equation (3-1) as a congruence modulo $\eta_{4} \eta_{7}^{2}$. For this, we replace $\eta_{10}$ by its value given by the equation (3-1) in the height conditions (3-8) and (3-11). These conditions become

$$
\begin{aligned}
&\left|\eta_{8} \eta_{9}\right|\left|\eta_{2} \eta_{5}^{2} \eta_{8}+\eta_{3} \eta_{6}^{2} \eta_{9}-\eta_{1} \eta_{2} \eta_{3} \eta_{4} \eta_{5} \eta_{6} \eta_{7}\right| \leq B \eta_{4} \eta_{7}^{2}, \\
& \eta_{1}^{2} \eta_{2} \eta_{3} \eta_{4}\left|\eta_{2} \eta_{5}^{2} \eta_{8}+\eta_{3} \eta_{6}^{2} \eta_{9}-\eta_{1} \eta_{2} \eta_{3} \eta_{4} \eta_{5} \eta_{6} \eta_{7}\right| \leq B,
\end{aligned}
$$

and we still denote them respectively by (3-8) and (3-11). From now on, we use the notation $\eta=\left(\eta_{2}, \eta_{3}, \eta_{4}, \eta_{5}, \eta_{6}, \eta_{7}\right)$, and we set

$$
\boldsymbol{\eta}^{\left(r_{2}, r_{3}, r_{4}, r_{5}, r_{6}, r_{7}\right)}=\eta_{2}^{r_{2}} \eta_{3}^{r_{3}} \eta_{4}^{r_{4}} \eta_{5}^{r_{5}} \eta_{6}^{r_{6}} \eta_{7}^{r_{7}}
$$

for $\left(r_{2}, r_{3}, r_{4}, r_{5}, r_{6}, r_{7}\right) \in \mathbb{Q}^{6}$. We set

$$
Y=\frac{B}{\eta_{2} \eta_{3} \eta_{4}}, \quad Z_{1}=\frac{B^{1 / 3}}{\eta^{(2 / 3,2 / 3,2 / 3,1 / 3,1 / 3,1 / 3)}},
$$

and, for brevity, $q_{8}=\eta_{2} \eta_{5}^{2}, q_{9}=\eta_{3} \eta_{6}^{2}, q_{10}=\eta_{4} \eta_{7}^{2}$. It is immediate to check that $\eta$ is restricted to lie in the region $\mathscr{V}$ defined by

$$
\begin{aligned}
\mathscr{V}=\left\{\eta \in \mathbb{Z}_{>0}^{6} \mid Y(\log \log B)^{2 / 3} \geq q_{8} Z_{1}^{2},\right. & Y(\log \log B)^{2 / 3} \geq q_{9} Z_{1}^{2}, \\
& \left.Z_{1} \geq 3^{-1 / 3}, q_{8} \geq q_{10}, q_{9} \geq q_{10}\right\} .
\end{aligned}
$$

We fix $\eta_{1} \in \mathbb{Z}_{>0}$ and $\eta \in \mathscr{V}$, subject to the conditions (5-15), (5-17) and (5-18) and to the coprimality conditions (3-5)-(3-7). Let $N\left(\eta_{1}, \eta, B\right)$ be the number of $\left(\eta_{8}, \eta_{9}, \eta_{10}\right) \in \mathbb{Z}_{\neq 0}^{3}$ satisfying the equation (3-1), the height conditions (3-8)-(3-11), and finally the coprimality conditions (3-2)-(3-4). The goal of this section is to prove the following lemma: 
Lemma 16. We have the estimate

$$
N\left(\eta_{1}, \boldsymbol{\eta}, B\right)=\frac{B^{2 / 3}}{\boldsymbol{\eta}^{(1 / 3,1 / 3,1 / 3,2 / 3,2 / 3,2 / 3)}} g_{2}\left(\frac{\eta_{1}}{Z_{1}}\right) \theta_{1}\left(\eta_{1}, \boldsymbol{\eta}\right) \theta_{2}(\boldsymbol{\eta})+R\left(\eta_{1}, \boldsymbol{\eta}, B\right),
$$

where $\theta_{1}\left(\eta_{1}, \boldsymbol{\eta}\right)$ and $\theta_{2}(\boldsymbol{\eta})$ are arithmetic functions defined in (5-28) and (5-29) respectively and

$$
\sum_{\eta_{1}, \eta} R\left(\eta_{1}, \boldsymbol{\eta}, B\right) \ll B(\log B)^{5}(\log \log B)^{7 / 3} .
$$

First, we see that since $\operatorname{gcd}\left(\eta_{2} \eta_{5}, \eta_{3} \eta_{6} \eta_{9}\right)=1$ and $\operatorname{gcd}\left(\eta_{3} \eta_{6}, \eta_{2} \eta_{5} \eta_{8}\right)=1$, the equation (3-1) proves that the coprimality condition (3-2) can be replaced by $\operatorname{gcd}\left(\eta_{10}, \eta_{1} \eta_{4}\right)=1$. Let us remove the coprimality conditions $\operatorname{gcd}\left(\eta_{8}, \eta_{6}\right)=1$ and $\operatorname{gcd}\left(\eta_{9}, \eta_{5}\right)=1$ using Möbius inversions; we obtain

$$
\begin{aligned}
& N\left(\eta_{1}, \boldsymbol{\eta}, B\right)=\sum_{k_{8} \mid \eta_{6}} \mu\left(k_{8}\right) \quad \sum_{k_{9} \mid \eta_{5}} \mu\left(k_{9}\right) S_{k_{8}, k_{9}}\left(\eta_{1}, \boldsymbol{\eta}, B\right), \\
& \operatorname{gcd}\left(k_{8}, \eta_{1} \eta_{2} \eta_{3} \eta_{4} \eta_{7}\right)=1 \quad \operatorname{gcd}\left(k_{9}, \eta_{1} \eta_{2} \eta_{3} \eta_{4} \eta_{7}\right)=1
\end{aligned}
$$

where $S_{k_{8}, k_{9}}\left(\eta_{1}, \boldsymbol{\eta}, B\right)$ is the cardinality of

$$
\begin{array}{r}
\left\{\left(\eta_{8}^{\prime}, \eta_{9}^{\prime}, \eta_{10}\right) \in \mathbb{Z}_{\neq 0}^{3} \mid \eta_{2} \eta_{5}^{2} k_{8} \eta_{8}^{\prime}+\eta_{3} \eta_{6}^{2} k_{9} \eta_{9}^{\prime}+\eta_{4} \eta_{7}^{2} \eta_{10}=b, \operatorname{gcd}\left(\eta_{10}, \eta_{1} \eta_{4}\right)=1,\right. \\
\left.(3-8),(3-9),(3-10),(3-11), \operatorname{gcd}\left(\eta_{8}^{\prime} \eta_{9}^{\prime}, \eta_{1} \eta_{2} \eta_{3} \eta_{4} \eta_{7}\right)=1\right\},
\end{array}
$$

and where we use the notation $\eta_{8}=k_{8} \eta_{8}^{\prime}, \eta_{9}=k_{9} \eta_{9}^{\prime}$ and $b=\eta_{1} \eta_{2} \eta_{3} \eta_{4} \eta_{5} \eta_{6} \eta_{7}$.

From now on, we set

$$
\mathscr{Z}=B^{1 / \log \log B} .
$$

To take care of the error terms showing up in the application of Lemma 7, we need to show that the summations over $k_{8}$ and $k_{9}$ can be restricted to $k_{8}, k_{9} \leq \mathscr{L}^{3}$. To do so, let $N^{\prime}\left(\eta_{1}, \eta, B\right)$ be the contribution of $N\left(\eta_{1}, \boldsymbol{\eta}, B\right)$ under the assumption $k_{8}>\mathscr{L}^{3}$; that is,

$$
N^{\prime}\left(\eta_{1}, \boldsymbol{\eta}, B\right)=\sum_{\substack{k_{8} \mid \eta_{6}, k_{8}>o_{\mathcal{L}^{3}} \\ \operatorname{gcd}\left(k_{8}, \eta_{1} \eta_{2} \eta_{3} \eta_{4} \eta_{7}\right)=1}} \sum_{\substack{k_{9} \mid \eta_{5} \\ \operatorname{gcd}\left(k_{9}, \eta_{1} \eta_{2} \eta_{3} \eta_{4} \eta_{7}\right)=1}} S_{k_{8}, k_{9}}\left(\eta_{1}, \boldsymbol{\eta}, B\right) .
$$

Let us write $\eta_{6}=k_{8} \eta_{6}^{\prime}$ and $\eta_{5}=k_{9} \eta_{5}^{\prime}$. We notice that the equation in the definition of $S_{k_{8}, k_{9}}\left(\eta_{1}, \boldsymbol{\eta}, B\right)$ implies that $k_{8} k_{9} \mid \eta_{10}$, and thus we also write $\eta_{10}=k_{8} k_{9} \xi_{10}$. With this notation, we get

$$
N^{\prime}\left(\eta_{1}, \boldsymbol{\eta}, B\right)=\sum_{\substack{\mathscr{q}^{3}<k_{8} \leq B^{1 / 2} \\ \operatorname{gcd}\left(k_{8}, \eta_{1} \eta_{2} \eta_{3} \eta_{4} \eta_{7}\right)=1}} \sum_{\substack{k_{9} \leq B^{1 / 2} \\ \operatorname{gcd}\left(k_{9}, \eta_{1} \eta_{2} \eta_{3} \eta_{4} \eta_{7}\right)=1}} S_{k_{8}, k_{9}}^{\prime}\left(\eta_{1}, \boldsymbol{\eta}, B\right),
$$

where $S_{k_{8}, k_{9}}^{\prime}\left(\eta_{1}, \boldsymbol{\eta}, B\right)$ is the cardinality of 


$$
\begin{aligned}
\left\{\left(\eta_{8}^{\prime}, \eta_{9}^{\prime}, \xi_{10}\right) \in \mathbb{Z}_{\neq 0}^{3} \mid \eta_{2} \eta_{5}^{\prime 2} k_{9} \eta_{8}^{\prime}+\eta_{3} \eta_{6}^{\prime 2} k_{8} \eta_{9}^{\prime}+\eta_{4} \eta_{7}^{2} \xi_{10}=b^{\prime}, \operatorname{gcd}\left(\xi_{10}, \eta_{1} \eta_{4}\right)\right. & =1, \\
(3-8),(3-9),(3-10),(3-11), \operatorname{gcd}\left(\eta_{8}^{\prime} \eta_{9}^{\prime}, \eta_{1} \eta_{2} \eta_{3} \eta_{4} \eta_{7}\right) & =1\},
\end{aligned}
$$

where we have set $b^{\prime}=\eta_{1} \eta_{2} \eta_{3} \eta_{4} \eta_{5}^{\prime} \eta_{6}^{\prime} \eta_{7}$. Let us split the summations over $k_{8}$ and $k_{9}$ into dyadic ranges. Let us assume that $K_{8}, K_{9} \geq \frac{1}{2}$ and that $K_{8}<k_{8} \leq 2 K_{8}$ and $K_{9}<k_{9} \leq 2 K_{9}$. Let us set $\xi_{8}=k_{9} \eta_{8}^{\prime}$ and $\xi_{9}=k_{8} \eta_{9}^{\prime}$. The height conditions (3-8), (3-9), (3-10) and (3-11) imply respectively

$$
\begin{aligned}
\left|\xi_{8} \xi_{9} \xi_{10}\right| & \leq \frac{B}{K_{8} K_{9}}, \\
\eta_{1}^{2} \eta_{2}^{2} \eta_{3} \eta_{4} \eta_{5}^{\prime 2}\left|\xi_{8}\right| & \leq \frac{B}{K_{8} K_{9}}, \\
\eta_{1}^{2} \eta_{2} \eta_{3}^{2} \eta_{4} \eta_{6}^{\prime 2}\left|\xi_{9}\right| & \leq \frac{B}{K_{8} K_{9}}, \\
\eta_{1}^{2} \eta_{2} \eta_{3} \eta_{4}^{2} \eta_{7}^{2}\left|\xi_{10}\right| & \leq \frac{B}{K_{8} K_{9}} .
\end{aligned}
$$

We thus have, for $K_{8}<k_{8} \leq 2 K_{8}$ and $K_{9}<k_{9} \leq 2 K_{9}$,

$S_{k_{8}, k_{9}}^{\prime}\left(\eta_{1}, \eta, B\right)$

$\ll \#\left\{\left(\xi_{8}, \xi_{9}, \xi_{10}\right) \in \mathbb{Z}_{\neq 0}^{3}\left|k_{8}\right| \xi_{9}, k_{9} \mid \xi_{8}, \eta_{2} \eta_{5}^{\prime 2} \xi_{8}+\eta_{3} \eta_{6}^{\prime 2} \xi_{9}+\eta_{4} \eta_{7}^{2} \xi_{10}=b^{\prime}\right.$,

(5-21), (5-22), (5-23), (5-24), $\left.\operatorname{gcd}\left(\xi_{10}, \eta_{1} \eta_{4}\right)=1, \operatorname{gcd}\left(\xi_{8} \xi_{9}, \eta_{1} \eta_{2} \eta_{3} \eta_{4} \eta_{7}\right)=1\right\}$.

Therefore, using the standard bound for the divisor function,

$$
\tau(n) \ll n^{1 / \log \log (3 n)},
$$

for $n \geq 1$, we get

$$
\sum_{\substack{K_{8}<k_{8} \leq 2 K_{8} \\ K_{9}<k_{9} \leq 2 K_{9}}} S_{k_{8}, k_{9}}^{\prime}\left(\eta_{1}, \boldsymbol{\eta}, B\right) \ll \mathscr{L}^{2} S_{K_{8}, K_{9}},
$$

where $S_{K_{8}, K_{9}}=S_{K_{8}, K_{9}}\left(\eta_{1}, \eta_{2}, \eta_{3}, \eta_{4}, \eta_{5}^{\prime}, \eta_{6}^{\prime}, \eta_{7}, B\right)$ is the cardinality of $\left\{\left(\xi_{8}, \xi_{9}, \xi_{10}\right) \in \mathbb{Z}_{\neq 0}^{3} \mid \eta_{2} \eta_{5}^{\prime 2} \xi_{8}+\eta_{3} \eta_{6}^{\prime 2} \xi_{9}+\eta_{4} \eta_{7}^{2} \xi_{10}=b^{\prime},(5-21),(5-22),(5-23),(5-24)\right.$, $\left.\operatorname{gcd}\left(\xi_{10}, \eta_{1} \eta_{4}\right)=1, \operatorname{gcd}\left(\xi_{8} \xi_{9}, \eta_{1} \eta_{2} \eta_{3} \eta_{4} \eta_{7}\right)=1\right\}$.

Setting $\xi_{6,8}=\operatorname{gcd}\left(\eta_{6}^{\prime}, \xi_{8}\right)$ and $\xi_{5,9}=\operatorname{gcd}\left(\eta_{5}^{\prime}, \xi_{9}\right)$, we see that $\xi_{6,8} \xi_{5,9} \mid \xi_{10}$, and we thus obtain

$$
\sum_{\eta_{1}, \eta_{2}, \eta_{3}, \eta_{4}, \eta_{5}^{\prime}, \eta_{6}^{\prime}, \eta_{7}} S_{K_{8}, K_{9}} \ll \sum_{\xi_{6,8}, \xi_{5,9} \leq B} N_{U, H}\left(\frac{B}{K_{8} K_{9} \xi_{6,8} \xi_{5,9}}\right) .
$$


Therefore, we can apply [Browning 2006]. We get

$$
\sum_{\eta_{1}, \eta} N^{\prime}\left(\eta_{1}, \eta, B\right) \ll \mathscr{L}^{2} \sum_{\substack{\mathscr{L}^{3}<K_{8}<B^{1 / 2} \\ K_{9}<B^{1 / 2}}} \sum_{\xi_{6,8}, \xi_{5,9} \leq B} \frac{B(\log B)^{6}}{K_{8} K_{9} \xi_{6,8} \xi_{5,9}} \ll B \mathscr{L}^{-1 / 2}
$$

which is satisfactory. Therefore, we can restrict from now on the summations over $k_{8}$ and $k_{9}$ as we wished.

We note that if we allow $\eta_{10}=0$ in the definition of the cardinality $S_{k_{8}, k_{9}}\left(\eta_{1}, \eta, B\right)$ then the coprimality condition $\operatorname{gcd}\left(\eta_{10}, \eta_{1} \eta_{4}\right)=1$ implies $\eta_{1}=\eta_{4}=1$. Moreover, the equation $\eta_{2} \eta_{5}^{2} k_{8} \eta_{8}^{\prime}+\eta_{3} \eta_{6}^{2} k_{9} \eta_{9}^{\prime}=\eta_{2} \eta_{3} \eta_{5} \eta_{6} \eta_{7}$ also implies $\eta_{2}=\eta_{3}=1$. These restrictions are in contradiction with the condition (5-17), so from now on, we allow $\eta_{10}$ to vanish in the definition of $S_{k_{8}, k_{9}}\left(\eta_{1}, \eta, B\right)$. Let us now remove the coprimality condition $\operatorname{gcd}\left(\eta_{10}, \eta_{1} \eta_{4}\right)=1$ using a Möbius inversion. We get that the main term of $N\left(\eta_{1}, \boldsymbol{\eta}, B\right)$ is equal to

$$
\sum_{\substack{k_{8} \mid \eta_{6}, k_{8} \leq \mathscr{E}^{3} \\ \operatorname{gcd}\left(k_{8}, \eta_{1} \eta_{2} \eta_{3} \eta_{4} \eta_{7}\right)=1}} \mu\left(k_{8}\right) \sum_{\substack{k_{9} \mid \eta_{5}, k_{9} \leq \mathscr{E}^{3} \\ \operatorname{gcd}\left(k_{9}, \eta_{1} \eta_{2} \eta_{3} \eta_{4} \eta_{7}\right)=1}} \mu\left(k_{9}\right) \sum_{k_{10} \mid \eta_{1} \eta_{4}} \mu\left(k_{10}\right) S_{k_{8}, k_{9}, k_{10}}\left(\eta_{1}, \boldsymbol{\eta}, B\right),
$$

where $S_{k_{8}, k_{9}, k_{10}}\left(\eta_{1}, \boldsymbol{\eta}, B\right)$ denotes the cardinality of

$$
\begin{aligned}
\left\{\left(\eta_{8}^{\prime}, \eta_{9}^{\prime}, \eta_{10}^{\prime}\right) \in \mathbb{Z}_{\neq 0}^{2} \times \mathbb{Z} \mid\right. & \eta_{2} \eta_{5}^{2} k_{8} \eta_{8}^{\prime}+\eta_{3} \eta_{6}^{2} k_{9} \eta_{9}^{\prime}+\eta_{4} \eta_{7}^{2} k_{10} \eta_{10}^{\prime}=b, \\
& \left.(3-8),(3-9),(3-10),(3-11), \operatorname{gcd}\left(\eta_{8}^{\prime} \eta_{9}^{\prime}, \eta_{1} \eta_{2} \eta_{3} \eta_{4} \eta_{7}\right)=1\right\} .
\end{aligned}
$$

Since $\operatorname{gcd}\left(\eta_{1} \eta_{4}, k_{8} k_{9} \eta_{5} \eta_{6} \eta_{8}^{\prime} \eta_{9}^{\prime}\right)=1$, we have $\operatorname{gcd}\left(k_{10}, k_{8} k_{9} \eta_{5} \eta_{6} \eta_{8}^{\prime} \eta_{9}^{\prime}\right)=1$. Also, the two conditions $\operatorname{gcd}\left(\eta_{2} \eta_{5} k_{8} \eta_{8}^{\prime}, \eta_{3}\right)=1$ and $\operatorname{gcd}\left(\eta_{3} \eta_{6} k_{9} \eta_{9}^{\prime}, \eta_{2}\right)=1$ imply that we also have $\operatorname{gcd}\left(k_{10}, \eta_{2} \eta_{3}\right)=1$. We now remove the coprimality conditions $\operatorname{gcd}\left(\eta_{8}^{\prime} \eta_{9}^{\prime}, \eta_{1} \eta_{2} \eta_{3}\right)=1$ using Möbius inversions. Setting $\eta_{8}^{\prime}=\ell_{8} \eta_{8}^{\prime \prime}$ and $\eta_{9}^{\prime}=\ell_{9} \eta_{9}^{\prime \prime}$, we obtain that the main term of $N\left(\eta_{1}, \eta, B\right)$ is equal to

$$
\sum_{k_{8} \mid \eta_{6}, k_{8} \leq \mathscr{L}^{3}} \mu\left(k_{8}\right) \sum_{k_{9} \mid \eta_{5}, k_{9} \leq \mathscr{L}^{3}} \mu\left(k_{9}\right)
$$

$\operatorname{gcd}\left(k_{8}, \eta_{1} \eta_{2} \eta_{3} \eta_{4} \eta_{7}\right)=1 \quad \operatorname{gcd}\left(k_{9}, \eta_{1} \eta_{2} \eta_{3} \eta_{4} \eta_{7}\right)=1$

$$
\times \sum_{\substack{k_{10} \mid \eta_{1} \eta_{4} \\
\operatorname{gcd}\left(k_{10}, k_{8} k_{9} \eta_{2} \eta_{3} \eta_{5} \eta_{6}\right)=1}} \mu\left(k_{10}\right) \sum_{\begin{array}{c}
\ell_{8}, \ell_{9} \mid \eta_{1} \eta_{2} \eta_{3} \\
\operatorname{gcd}\left(\ell_{8} \ell_{9}, k_{10} \eta_{4} \eta_{7}\right)=1
\end{array}} \mu\left(\ell_{8}\right) \mu\left(\ell_{9}\right) S\left(\eta_{1}, \boldsymbol{\eta}, B\right),
$$

where $S\left(\eta_{1}, \eta, B\right)$ denotes the cardinality of

$$
\left\{\left(\eta_{8}^{\prime \prime}, \eta_{9}^{\prime \prime}\right) \in \mathbb{Z}_{\neq 0}^{2} \mid \eta_{2} \eta_{5}^{2} k_{8} \ell_{8} \eta_{8}^{\prime \prime}+\eta_{3} \eta_{6}^{2} k_{9} \ell_{9} \eta_{9}^{\prime \prime} \equiv b\left(\bmod k_{10} \eta_{4} \eta_{7}^{2}\right),\right.
$$

(3-8), (3-9), (3-10), (3-11), $\left.\operatorname{gcd}\left(\eta_{8}^{\prime \prime} \eta_{9}^{\prime \prime}, k_{10} \eta_{4} \eta_{7}\right)=1\right\}$. 
Note that we have replaced the equation $\eta_{2} \eta_{5}^{2} k_{8} \ell_{8} \eta_{8}^{\prime \prime}+\eta_{3} \eta_{6}^{2} k_{9} \ell_{9} \eta_{9}^{\prime \prime}+\eta_{4} \eta_{7}^{2} k_{10} \eta_{10}^{\prime}=b$ by a congruence.

Setting

$$
X=\frac{B}{\eta_{1}^{2} \boldsymbol{\eta}^{(1,1,1,0,0,0)}}, \quad T=\eta_{1} \boldsymbol{\eta}^{(1,1,1,1,1,1)}
$$

and $A_{1}=k_{8} \ell_{8} \eta_{2} \eta_{5}^{2}, A_{2}=k_{9} \ell_{9} \eta_{3} \eta_{6}^{2}$ and recalling the equality (2-21), it is immediate to check that $\left(\eta_{8}^{\prime \prime}, \eta_{9}^{\prime \prime}\right) \in \mathbb{Z}_{\neq 0}^{2}$ is subject to the height conditions (3-8)-(3-11) if and only if $\left(\eta_{8}^{\prime \prime}, \eta_{9}^{\prime \prime}\right) \in \mathscr{Y} \cap \mathbb{Z}_{\neq 0}^{2}$. Setting $\mathscr{L}=\log \log B$, we see that the condition (5-18) can be rewritten $X / \mathscr{L} \leq T$. We can therefore apply Lemma 7 with $L=\log B$, $q=k_{10} \eta_{4} \eta_{7}^{2}$ and $\boldsymbol{a}=\left(k_{8} \ell_{8} \eta_{2} \eta_{5}^{2}, k_{9} \ell_{9} \eta_{3} \eta_{6}^{2}\right)$. Recall the definitions (2-24) of $\varphi^{*}$ and (5-19) of $Z_{1}$ and also the definitions of $E(q, a)$ and $E_{2}(q)$, given in Lemmas 2 and 6 respectively. We obtain

$$
S\left(\eta_{1}, \boldsymbol{\eta}, B\right)-\frac{\varphi^{*}\left(k_{10} \eta_{4} \eta_{7}\right)}{k_{8} \ell_{8} k_{9} \ell_{9} k_{10}} \frac{B^{2 / 3}}{\eta^{(1 / 3,1 / 3,1 / 3,2 / 3,2 / 3,2 / 3)}} g_{2}\left(\frac{\eta_{1}}{Z_{1}}\right) \ll \mathscr{E}+\mathscr{E}^{\prime},
$$

where

$$
\mathscr{E}=(\log B)^{6} E(q, \boldsymbol{a})
$$

and

$$
\begin{aligned}
& \mathscr{E}^{\prime}=\frac{B^{2 / 3}}{k_{8} \ell_{8} k_{9} \ell_{9} k_{10} \eta^{(1 / 3,1 / 3,1 / 3,2 / 3,2 / 3,2 / 3)}} \mathscr{L}^{4 / 3} \\
& \quad \times\left(\frac{\mathscr{L}}{\log B}+\frac{k_{8}^{1 / 2} \ell_{8}^{1 / 2} \eta_{1} \eta_{2} \eta_{3}^{1 / 2} \eta_{4}^{1 / 2} \eta_{5}}{B^{1 / 2}}+\frac{k_{9}^{1 / 2} \ell_{9}^{1 / 2} \eta_{1} \eta_{2}^{1 / 2} \eta_{3} \eta_{4}^{1 / 2} \eta_{6}}{B^{1 / 2}}\right) E_{2}(q) .
\end{aligned}
$$

Let us estimate the contribution of these error terms. Let us start by bounding the overall contribution of $\mathscr{E}$. For this, we write $\eta_{5}=k_{9} \eta_{5}^{\prime}$ and $\eta_{6}=k_{8} \eta_{6}^{\prime}$, and we let $Y_{5}, Y_{6}$ and $Y_{7}$ be variables running over the set $\left\{2^{n} \mid n \geq-1\right\}$. We define $\mathcal{N}=\mathcal{N}\left(Y_{5}, Y_{6}, Y_{7}\right)$ as the sum over $\eta_{5}^{\prime}, \eta_{6}^{\prime}, \eta_{7} \in \mathbb{Z}_{\geq 1}$ satisfying $Y_{5}<k_{9} \eta_{5}^{\prime} \leq 2 Y_{5}, Y_{6}<$ $k_{8} \eta_{6}^{\prime} \leq 2 Y_{6}$ and $Y_{7}<\eta_{7} \leq 2 Y_{7}$ and the coprimality conditions $\operatorname{gcd}\left(\eta_{5}^{\prime} \eta_{6}^{\prime}, \eta_{4} \eta_{7}\right)=1$ and $\operatorname{gcd}\left(\eta_{5}^{\prime}, \eta_{6}^{\prime}\right)=1$, of the quantity

$$
\sum_{\substack{k_{8}, k_{9} \leq \mathscr{L}^{3} \\
\operatorname{gcd}\left(k_{8} k_{9}, \eta_{1} \eta_{2} \eta_{3} \eta_{4} \eta_{7}\right)=1}} \sum_{\substack{k_{10} \mid \eta_{1} \eta_{4} \\
\operatorname{gcd}\left(k_{10}, k_{8} k_{9} \eta_{2} \eta_{3} \eta_{5}^{\prime} \eta_{6}^{\prime}\right)=1}} \sum_{\begin{array}{c}
\ell_{8}, \ell_{9} \mid \eta_{1} \eta_{2} \eta_{3} \\
\operatorname{gcd}\left(\ell_{8} \ell_{9}, k_{10} \eta_{4} \eta_{7}\right)=1
\end{array}}(\log B)^{6} E\left(q, \boldsymbol{a}^{\prime}\right),
$$

where $\boldsymbol{a}^{\prime}=\left(k_{9} \ell_{8} \eta_{2} \eta_{5}^{\prime 2}, k_{8} \ell_{9} \eta_{3} \eta_{6}^{\prime 2}\right)$. We now aim to bound the contribution of the error term $\mathscr{E}$ by first estimating the quantity $\mathcal{N}$ and then by summing $\mathcal{N}$ over $\eta_{1}, \eta_{2}$, $\eta_{3}$ and $\eta_{4}$ and over all the possible values for $Y_{5}, Y_{6}$ and $Y_{7}$. Note that the variables 
$Y_{5}, Y_{6}$ and $Y_{7}$ satisfy the inequalities

$$
\begin{aligned}
\eta_{1}^{3} \eta_{2}^{2} \eta_{3}^{2} \eta_{4}^{2} Y_{5} Y_{6} Y_{7} & \leq 3 B, \\
\eta_{4} Y_{7}^{2} & \leq 4 \eta_{2} Y_{5}^{2}, \\
\eta_{4} Y_{7}^{2} & \leq 4 \eta_{3} Y_{6}^{2} .
\end{aligned}
$$

Applying Lemma 9 to sum over $\eta_{5}^{\prime}$ and $\eta_{6}^{\prime}$ and recalling that $q=k_{10} \eta_{4} \eta_{7}^{2}$, we see that

$$
\begin{aligned}
\mathcal{N} & \ll(\log B)^{6} \sum_{Y_{7}<\eta_{7} \leq 2 Y_{7}} \sum_{k_{8}, k_{9} \leq \mathscr{L}^{3}} \sum_{k_{10} \mid \eta_{1} \eta_{4}} \sum_{\ell_{8}, \ell_{9} \mid \eta_{1} \eta_{2} \eta_{3}}\left(\frac{Y_{5} Y_{6}}{k_{8} k_{9}}+k_{10} \eta_{4} \eta_{7}^{2}\right) \tau(q)^{2} E_{1}(q) \\
& \ll \mathscr{L}^{7} \sum_{Y_{7}<\eta_{7} \leq 2 Y_{7}} \tau\left(\eta_{1} \eta_{4}\right) \tau\left(\eta_{1} \eta_{2} \eta_{3}\right)^{2} \tau\left(\eta_{1} \eta_{4}^{2} \eta_{7}^{2}\right)^{2}\left(Y_{5} Y_{6}+\eta_{1} \eta_{4}^{2} \eta_{7}^{2}\right) \\
& \ll \mathscr{L}^{12}\left(Y_{5} Y_{6} Y_{7}+\eta_{1} \eta_{4}^{2} Y_{7}^{3}\right) .
\end{aligned}
$$

Using the two conditions (5-26) and (5-27), we finally obtain

$$
\mathcal{N} \ll \mathscr{L}^{12} \eta_{1} \eta_{2}^{1 / 2} \eta_{3}^{1 / 2} \eta_{4} Y_{5} Y_{6} Y_{7}
$$

We now aim to sum this quantity over all the possible values for $Y_{5}, Y_{6}$ and $Y_{7}$. Let us start by summing over $Y_{7}$ using the condition (5-25) and then over $\eta_{1}$ using the condition (5-17); we obtain

$$
\begin{aligned}
\sum_{Y_{i}} \mathcal{N} & \ll \mathscr{L}^{12} \sum_{\eta_{1}, \eta_{2}, \eta_{3}, \eta_{4}, Y_{5}, Y_{6}, Y_{7}} \eta_{1} \eta_{2}^{1 / 2} \eta_{3}^{1 / 2} \eta_{4} Y_{5} Y_{6} Y_{7} \\
& \ll B \mathscr{L}^{13} \sum_{\eta_{1}, \eta_{2}, \eta_{3}, \eta_{4}} \frac{1}{\eta_{1}^{2} \eta_{2}^{3 / 2} \eta_{3}^{3 / 2} \eta_{4}} \ll B \mathscr{L}^{-2} \sum_{\eta_{2}, \eta_{3}, \eta_{4}} \frac{\mathrm{sq}\left(\eta_{2} \eta_{3} \eta_{4}\right)}{\eta_{2}^{3 / 2} \eta_{3}^{3 / 2} \eta_{4}} \\
& \ll B \mathscr{L}^{-1},
\end{aligned}
$$

which is satisfactory. In addition, the overall contributions of the three terms of the error term $\mathscr{C}^{\prime}$ are easily seen to be bounded by $B(\log B)^{5}(\log \log B)^{7 / 3}$, which is also satisfactory.

Therefore, the main term of $N\left(\eta_{1}, \boldsymbol{\eta}, B\right)$ is equal to

$$
\sum_{\substack{k_{8} \mid \eta_{6}, k_{8} \leq \mathscr{L}^{3} \\ \operatorname{gcd}\left(k_{8}, \eta_{1} \eta_{2} \eta_{3} \eta_{4} \eta_{7}\right)=1}} \frac{\mu\left(k_{8}\right)}{k_{8}} \sum_{\substack{k_{9} \mid \eta_{5}, k_{9} \leq \mathfrak{L}^{3} \\ \operatorname{gcd}\left(k_{9}, \eta_{1} \eta_{2} \eta_{3} \eta_{4} \eta_{7}\right)=1}} \frac{\mu\left(k_{9}\right)}{k_{9}} \sum_{\substack{k_{10} \mid \eta_{1} \eta_{4} \\ \operatorname{gcd}\left(k_{10}, k_{8} k_{9} \eta_{2} \eta_{3} \eta_{5} \eta_{6}\right)=1}} \frac{\mu\left(k_{10}\right)}{k_{10}}
$$

$$
\times \sum_{\substack{\ell_{8}, \ell_{9} \mid \eta_{1} \eta_{2} \eta_{3} \\ \operatorname{gcd}\left(\ell_{8} \ell_{9}, k_{10} \eta_{4} \eta_{7}\right)=1}} \frac{\mu\left(\ell_{8}\right)}{\ell_{8}} \frac{\mu\left(\ell_{9}\right)}{\ell_{9}} \varphi^{*}\left(k_{10} \eta_{4} \eta_{7}\right) \frac{B^{2 / 3}}{\eta^{(1 / 3,1 / 3,1 / 3,2 / 3,2 / 3,2 / 3)}} g_{2}\left(\frac{\eta_{1}}{Z_{1}}\right) .
$$


Using the bound of Lemma 5 for $g_{2}$, we see that this quantity is

$$
\ll \sum_{\substack{k_{8}\left|\eta_{6}, k_{9}\right| \eta_{5} \\ k_{8}, k_{9} \leq \mathscr{L}^{3}}} \frac{1}{k_{8}} \frac{1}{k_{9}} \sigma_{-1}\left(\eta_{1} \eta_{4}\right) \sigma_{-1}\left(\eta_{1} \eta_{2} \eta_{3}\right)^{2} \frac{B^{2 / 3}}{\eta^{(1 / 3,1 / 3,1 / 3,2 / 3,2 / 3,2 / 3)}} .
$$

As a result, we see that if we remove the conditions $k_{8}, k_{9} \leq \mathscr{L}^{3}$ from the sums over $k_{8}$ and $k_{9}$, we create an error term whose overall contribution is, for instance, seen to be bounded by $B \mathscr{L}^{-1}$. Thus, we have proved that we can write

$$
N\left(\eta_{1}, \boldsymbol{\eta}, B\right)=M\left(\eta_{1}, \boldsymbol{\eta}, B\right)+R\left(\eta_{1}, \boldsymbol{\eta}, B\right),
$$

where

$$
\sum_{\eta_{1}, \eta} R\left(\eta_{1}, \eta, B\right) \ll B(\log B)^{5}(\log \log B)^{7 / 3}
$$

and

$$
M\left(\eta_{1}, \boldsymbol{\eta}, B\right)=\frac{B^{2 / 3}}{\boldsymbol{\eta}^{(1 / 3,1 / 3,1 / 3,2 / 3,2 / 3,2 / 3)}} g_{2}\left(\frac{\eta_{1}}{Z_{1}}\right) \theta\left(\eta_{1}, \boldsymbol{\eta}\right),
$$

where

$$
\begin{aligned}
& \theta\left(\eta_{1}, \boldsymbol{\eta}\right)= \sum_{\substack{k_{8} \mid \eta_{6} \\
\operatorname{gcd}\left(k_{8}, \eta_{1} \eta_{2} \eta_{3} \eta_{4} \eta_{7}\right)=1}} \frac{\mu\left(k_{8}\right)}{k_{8}} \sum_{\substack{k_{9} \mid \eta_{5} \\
\operatorname{gcd}\left(k_{9}, \eta_{1} \eta_{2} \eta_{3} \eta_{4} \eta_{7}\right)=1}} \frac{\mu\left(k_{9}\right)}{k_{9}} \\
& \times \sum_{\substack{k_{10} \mid \eta_{1} \eta_{4} \\
\operatorname{gcd}\left(k_{10}, k_{8} k_{9} \eta_{2} \eta_{3} \eta_{5} \eta_{6}\right)=1}} \frac{\mu\left(k_{10}\right)}{k_{10}} \sum_{\substack{\ell_{8}, \ell_{9} \mid \eta_{1} \eta_{2} \eta_{3} \\
\operatorname{gcd}\left(\ell_{8} \ell_{9}, k_{10} \eta_{4} \eta_{7}\right)=1}} \frac{\mu\left(\ell_{8}\right)}{\ell_{8}} \frac{\mu\left(\ell_{9}\right)}{\ell_{9}} \varphi^{*}\left(k_{10} \eta_{4} \eta_{7}\right) \\
&=\frac{\varphi^{*}\left(\eta_{3} \eta_{6}\right)}{\varphi^{*}\left(\eta_{3}\right)} \frac{\varphi^{*}\left(\eta_{2} \eta_{5}\right)}{\varphi^{*}\left(\eta_{2}\right)} \varphi^{*}\left(\eta_{1} \eta_{2} \eta_{3} \eta_{4} \eta_{7}\right)^{2} \sum_{\substack{k_{10} \mid \eta_{1} \eta_{4} \\
\operatorname{gcd}\left(k_{10}, \eta_{2} \eta_{3} \eta_{5} \eta_{6}\right)=1}} \frac{\mu\left(k_{10}\right)}{k_{10} \varphi^{*}\left(\eta_{4} \eta_{7} k_{10}\right)} .
\end{aligned}
$$

It is easy to check that for $a, b, c \geq 1$, we have

$$
\sum_{\substack{k \mid a \\ \operatorname{gcd}(k, c)=1}} \frac{\mu(k)}{k \varphi^{*}(k b)}=\frac{\varphi^{*}(\operatorname{gcd}(a, b))}{\varphi^{*}(b) \varphi^{*}(\operatorname{gcd}(a, b, c))} \prod_{\substack{p \mid a \\ p \nmid b c}}\left(1-\frac{1}{p-1}\right) .
$$

Using this equality and the remaining coprimality conditions (3-5), (3-6) and (3-7) and recalling the definition (2-26) of $\psi$, we see that we can write

$$
\theta\left(\eta_{1}, \boldsymbol{\eta}\right)=\theta_{1}\left(\eta_{1}, \boldsymbol{\eta}\right) \theta_{2}(\boldsymbol{\eta})
$$

where

$$
\theta_{1}\left(\eta_{1}, \boldsymbol{\eta}\right)=\psi_{\eta_{2} \eta_{3} \eta_{4}}\left(\eta_{1}\right)
$$

and

$$
\theta_{2}(\boldsymbol{\eta})=\varphi^{*}\left(\eta_{2} \eta_{3} \eta_{4}\right) \varphi^{*}\left(\eta_{2} \eta_{3} \eta_{4} \eta_{5} \eta_{6} \eta_{7}\right)
$$


5.4. Summation over $\eta_{\mathbf{1}}$. We now need to sum the main term of $N\left(\eta_{1}, \boldsymbol{\eta}, B\right)$ over $\eta_{1} \in \mathbb{Z}_{>0}$, where $\eta_{1}$ is subject to the conditions (5-17) and (5-18) (the condition (5-15) is implied by the definition of $g_{2}$ ) and to the coprimality condition (3-5). We start by proving that we can remove the restrictions that $\eta_{1}$ satisfies the conditions (5-17) and (5-18). Indeed, let us first assume that we have the condition

$$
\eta_{1} \operatorname{sq}\left(\eta_{2} \eta_{3} \eta_{4}\right)<B^{15 / \log \log B} .
$$

The bound of Lemma 5 for $g_{2}$ implies that the main term $M\left(\eta_{1}, \boldsymbol{\eta}, B\right)$ of $N\left(\eta_{1}, \boldsymbol{\eta}, B\right)$ satisfies

$$
M\left(\eta_{1}, \boldsymbol{\eta}, B\right) \ll \frac{B^{2 / 3}}{\boldsymbol{\eta}^{(1 / 3,1 / 3,1 / 3,2 / 3,2 / 3,2 / 3)}} .
$$

Let us now sum this quantity over $\eta_{7}$ using the condition (5-15) and then over $\eta_{1}$ using the condition (5-30); we obtain

$$
\begin{aligned}
\sum_{\eta_{1}, \boldsymbol{\eta}} M\left(\eta_{1}, \boldsymbol{\eta}, B\right) & \ll \sum_{\eta_{1}, \eta_{2}, \eta_{3}, \eta_{4}, \eta_{5}, \eta_{6}} \frac{B}{\eta_{1} \boldsymbol{\eta}^{(1,1,1,1,1,0)}} \\
& \ll \sum_{\eta_{2}, \eta_{3}, \eta_{4}, \eta_{5}, \eta_{6}} \frac{B(\log B)}{\boldsymbol{\eta}^{(1,1,1,1,1,0)} \log \log B} \ll \frac{B(\log B)^{6}}{\log \log B} .
\end{aligned}
$$

This error term is satisfactory. Let us now assume that we have the condition

$$
\eta_{1}^{3} \eta_{2}^{2} \eta_{3}^{2} \eta_{4}^{2} \eta_{5} \eta_{6} \eta_{7}<\frac{B}{\log \log B}
$$

Let us sum over $\eta_{1}$ using this condition; we get

$$
\sum_{\eta_{1}, \boldsymbol{\eta}} M\left(\eta_{1}, \boldsymbol{\eta}, B\right) \ll \sum_{\eta} \frac{B}{\boldsymbol{\eta}^{(1,1,1,1,1,1)}(\log \log B)^{1 / 3}} \ll \frac{B(\log B)^{6}}{(\log \log B)^{1 / 3}} .
$$

This error term is also satisfactory. We can thus remove the restrictions that $\eta_{1}$ satisfies the conditions (5-17) and (5-18), and we proceed to sum over $\eta_{1}$. Recall the definition (5-20) of $\mathscr{V}$. For fixed $\eta \in \mathscr{V}$ satisfying the coprimality conditions (3-6) and (3-7), let $N(\boldsymbol{\eta}, B)$ be the sum of the main term of $N\left(\eta_{1}, \boldsymbol{\eta}, B\right)$ over $\eta_{1}$, where $\eta_{1}$ is subject to the coprimality condition (3-5). Recall the definition (2-27) of $\Upsilon$. We now prove the following lemma.

Lemma 17. We have the estimate

$$
N(\boldsymbol{\eta}, B)=\Upsilon \frac{\omega_{\infty}}{3} \frac{B}{\eta^{(1,1,1,1,1,1)}} \Theta(\boldsymbol{\eta})+R(\boldsymbol{\eta}, B),
$$

where $\Theta(\eta)$ is a certain arithmetic function defined in (5-31) and where

$$
\sum_{\eta} R(\eta, B) \ll B(\log B)^{5} .
$$


Proof. Let us use Lemma 10 to sum over $\eta_{1}$. For any fixed $0<\gamma \leq 1$, we obtain

$$
\begin{aligned}
N(\boldsymbol{\eta}, B)=\Upsilon & \frac{B}{\boldsymbol{\eta}^{(1,1,1,1,1,1)}} \Theta(\boldsymbol{\eta}) \int_{t>0} g_{2}(t) \mathrm{d} t \\
& +O\left(\frac{B^{2 / 3}}{\boldsymbol{\eta}^{(1 / 3,1 / 3,1 / 3,2 / 3,2 / 3,2 / 3)}} Z_{1}^{\gamma} \sigma_{-\gamma / 2}\left(\eta_{2} \eta_{3} \eta_{4} \eta_{5} \eta_{6} \eta_{7}\right) \sup _{t>0} g_{2}(t)\right),
\end{aligned}
$$

where

$$
\Theta(\boldsymbol{\eta})=\varphi^{*}\left(\eta_{2} \eta_{3} \eta_{4}\right) \varphi^{*}\left(\eta_{2} \eta_{3} \eta_{4} \eta_{5} \eta_{6} \eta_{7}\right) \varphi^{*}\left(\eta_{5} \eta_{6} \eta_{7}\right) \varphi^{\curlyvee}\left(\eta_{2} \eta_{3} \eta_{4} \eta_{5} \eta_{6} \eta_{7}\right) .
$$

Let us set $\gamma=1 / 2$. Using the bound of Lemma 5 for $g_{2}$, we deduce that the overall contribution of this error term is

$$
\sum_{\eta} \frac{B^{5 / 6}}{\eta^{(2 / 3,2 / 3,2 / 3,5 / 6,5 / 6,5 / 6)}} \sigma_{-1 / 4}\left(\eta_{2} \eta_{3} \eta_{4} \eta_{5} \eta_{6} \eta_{7}\right) \ll B(\log B)^{5},
$$

where we have summed over $\eta$ using the condition $Z_{1} \geq 3^{-1 / 3}$. Recalling the definition of $g_{2}$ and the equality (4-1), we see that

$$
\int_{t>0} g_{2}(t) \mathrm{d} t=\frac{\omega_{\infty}}{3}
$$

which completes the proof.

5.5. Conclusion. It remains to sum the main term of $N(\boldsymbol{\eta}, B)$ over the $\boldsymbol{\eta} \in \mathcal{V}$ satisfying the coprimality conditions (3-6) and (3-7). It is easy to see that replacing $\mathscr{V}$ by the region

$$
\mathscr{V}^{\prime}=\left\{\eta \in \mathbb{Z}_{>0}^{6} \mid Y \geq q_{8} Z_{1}^{2}, Y \geq q_{9} Z_{1}^{2}, Z_{1} \geq 1, q_{8} \geq q_{10}, q_{9} \geq q_{10}\right\}
$$

produces an error term whose overall contribution is $\ll B(\log B)^{5} \log \log \log B$. Let us redefine the arithmetic function $\Theta$ as being equal to zero if the remaining coprimality conditions (3-6) and (3-7) are not satisfied. Recalling Lemma 15, we see that we have proved the following lemma:

Lemma 18. We have the estimate

$$
N_{U, H}(B)=\Upsilon \omega_{\infty} B \sum_{\eta \in \mathscr{V}^{\prime}} \frac{\Theta(\eta)}{\eta^{(1,1,1,1,1,1)}}+O\left(\frac{B(\log B)^{6}}{(\log \log B)^{1 / 6}}\right) .
$$

The end of the paper is dedicated to the completion of the proof of Theorem 1 . Let us introduce the generalized Möbius function $\mu$ defined for $\left(n_{1}, \ldots, n_{6}\right) \in \mathbb{Z}_{>0}^{6}$ by $\boldsymbol{\mu}\left(n_{1}, \ldots, n_{6}\right)=\mu\left(n_{1}\right) \cdots \mu\left(n_{6}\right)$. We set $\boldsymbol{k}=\left(k_{2}, k_{3}, k_{4}, k_{5}, k_{6}, k_{7}\right)$ and we define for $s \in \mathbb{C}$, such that $\Re(s)>1$, 
$F(s)=\sum_{\boldsymbol{\eta} \in \mathbb{Z}_{>0}^{6}} \frac{|(\Theta * \boldsymbol{\mu})(\boldsymbol{\eta})|}{\eta_{2}^{s} \eta_{3}^{s} \eta_{4}^{s} \eta_{5}^{s} \eta_{6}^{s} \eta_{7}^{s}}=\prod_{p}\left(\sum_{\boldsymbol{k} \in \mathbb{Z}_{\geq 0}^{6}} \frac{\left|(\Theta * \boldsymbol{\mu})\left(p^{k_{2}}, p^{k_{3}}, p^{k_{4}}, p^{k_{5}}, p^{k_{6}}, p^{k_{7}}\right)\right|}{p^{k_{2} s} p^{k_{3} s} p^{k_{4} s} p^{k_{5} s} p^{k_{6} s} p^{k_{7} s}}\right)$.

It is easy to check that if $\boldsymbol{k} \notin\{0,1\}^{6}$ then $(\Theta * \boldsymbol{\mu})\left(p^{k_{2}}, p^{k_{3}}, p^{k_{4}}, p^{k_{5}}, p^{k_{6}}, p^{k_{7}}\right)=0$ and if exactly one of the $k_{i}$ is equal to 1 , then $(\Theta * \mu)\left(p^{k_{2}}, p^{k_{3}}, p^{k_{4}}, p^{k_{5}}, p^{k_{6}}, p^{k_{7}}\right) \ll 1 / p$, so the local factors $F_{p}$ of $F$ satisfy

$$
F_{p}(s)=1+O\left(\frac{1}{p^{\min (\Re(s)+1,2 \Re(s))}}\right) .
$$

This proves that the function $F$ converges in the half-plane $\Re(s)>1 / 2$, which implies that $\Theta$ satisfies the assumption of [Le Boudec 2012b, Lemma 8]. The application of this lemma provides

$$
\sum_{\boldsymbol{\eta} \in \mathscr{V}^{\prime}} \frac{\Theta(\boldsymbol{\eta})}{\boldsymbol{\eta}^{(1,1,1,1,1,1)}}=\alpha\left(\sum_{\boldsymbol{\eta} \in \mathbb{Z}_{>0}^{6}} \frac{(\Theta * \boldsymbol{\mu})(\boldsymbol{\eta})}{\boldsymbol{\eta}^{(1,1,1,1,1,1)}}\right)(\log B)^{6}+O\left((\log B)^{5}\right),
$$

where $\alpha$ is the volume of the polytope defined in $\mathbb{R}^{6}$ by $t_{2}, t_{3}, t_{4}, t_{5}, t_{6}, t_{7} \geq 0$ and

$$
\begin{aligned}
2 t_{2}-t_{3}-t_{4}+4 t_{5}-2 t_{6}-2 t_{7} & \leq 1, \\
-t_{2}+2 t_{3}-t_{4}-2 t_{5}+4 t_{6}-2 t_{7} & \leq 1, \\
2 t_{2}+2 t_{3}+2 t_{4}+t_{5}+t_{6}+t_{7} & \leq 1, \\
-t_{2}+t_{4}-2 t_{5}+2 t_{7} & \leq 0, \\
-t_{3}+t_{4}-2 t_{6}+2 t_{7} & \leq 0 .
\end{aligned}
$$

It is easy to compute $\alpha$ using Franz's additional Maple package Convex [2009]. We find $\alpha=1 / 23040$; that is,

$$
\alpha=\alpha(\tilde{V}) .
$$

Furthermore, we have

$$
\begin{aligned}
\sum_{\boldsymbol{\eta} \in \mathbb{Z}_{>0}^{6}} \frac{(\Theta * \boldsymbol{\mu})(\boldsymbol{\eta})}{\boldsymbol{\eta}^{(1,1,1,1,1,1)}} & =\prod_{p}\left(\sum_{\boldsymbol{k} \in \mathbb{Z}_{\geq 0}^{6}} \frac{(\Theta * \boldsymbol{\mu})\left(p^{k_{2}}, p^{k_{3}}, p^{k_{4}}, p^{k_{5}}, p^{k_{6}}, p^{k_{7}}\right)}{p^{k_{2}} p^{k_{3}} p^{k_{4}} p^{k_{5}} p^{k_{6}} p^{k_{7}}}\right) \\
& =\prod_{p}\left(1-\frac{1}{p}\right)^{6}\left(\sum_{\boldsymbol{k} \in \mathbb{Z}_{\geq 0}^{6}} \frac{\Theta\left(p^{k_{2}}, p^{k_{3}}, p^{k_{4}}, p^{k_{5}}, p^{k_{6}}, p^{k_{7}}\right)}{p^{k_{2}} p^{k_{3}} p^{k_{4}} p^{k_{5}} p^{k_{6}} p^{k_{7}}}\right) .
\end{aligned}
$$

The calculation of these local factors is straightforward, and we find

$$
\sum_{\boldsymbol{k} \in \mathbb{Z}_{\geq 0}^{6}} \frac{\Theta\left(p^{k_{2}}, p^{k_{3}}, p^{k_{4}}, p^{k_{5}}, p^{k_{6}}, p^{k_{7}}\right)}{p^{k_{2}} p^{k_{3}} p^{k_{4}} p^{k_{5}} p^{k_{6}} p^{k_{7}}}=\varphi^{\curlyvee}(p)\left(1-\frac{1}{p}\right)\left(1+\frac{7}{p}+\frac{1}{p^{2}}\right) .
$$


We finally obtain

$$
\sum_{\boldsymbol{\eta} \in \mathbb{Z}_{>0}^{6}} \frac{(\Theta * \boldsymbol{\mu})(\boldsymbol{\eta})}{\boldsymbol{\eta}^{(1,1,1,1,1,1)}}=\Upsilon^{-1} \prod_{p}\left(1-\frac{1}{p}\right)^{7} \omega_{p} .
$$

Putting together the equalities (5-32)-(5-34) and Lemma 18 completes the proof of Theorem 1.

\section{Acknowledgments}

It is a great pleasure for the author to thank his supervisor Professor de la Bretèche for his advice during the preparation of this work. The author is also grateful to Professor Browning for his careful reading of an earlier version of the manuscript.

This work has benefited from the financial support of the ANR PEPR (Points Entiers Points Rationnels).

\section{References}

[Baier and Derenthal 2012] S. Baier and U. Derenthal, "Quadratic congruences on average and rational points on cubic surfaces", preprint, 2012. arXiv 1205.0373v2

[Batyrev and Tschinkel 1998a] V. V. Batyrev and Y. Tschinkel, "Manin's conjecture for toric varieties", J. Algebraic Geom. 7:1 (1998), 15-53. MR 2000c:11107 Zbl 0946.14009

[Batyrev and Tschinkel 1998b] V. V. Batyrev and Y. Tschinkel, "Tamagawa numbers of polarized algebraic varieties", pp. 299-340 in Nombre et répartition de points de hauteur bornée (Paris, 1996), edited by E. Peyre, Astérisque 251, Société Mathématique de France, Paris, 1998. MR 2000d:11090 Zbl 0926.11045

[de la Bretèche and Browning 2014] R. de la Bretèche and T. D. Browning, "Density of Châtelet surfaces failing the Hasse principle", Proc. Lond. Math. Soc. (3) 108:4 (2014), 1030-1078. MR 3198755 Zbl 1291.14041

[de la Bretèche and Swinnerton-Dyer 2007] R. de la Bretèche and P. Swinnerton-Dyer, "Fonction zêta des hauteurs associée à une certaine surface cubique”, Bull. Soc. Math. France 135:1 (2007), 65-92. MR 2009f:14041 Zbl 1207.11068

[de la Bretèche et al. 2007] R. de la Bretèche, T. D. Browning, and U. Derenthal, "On Manin's conjecture for a certain singular cubic surface”, Ann. Sci. École Norm. Sup. (4) 40:1 (2007), 1-50. MR 2008e:11038 Zbl 1125.14008

[Browning 2006] T. D. Browning, "The density of rational points on a certain singular cubic surface", J. Number Theory 119:2 (2006), 242-283. MR 2007d:14046 Zbl 1119.11034

[Browning and Derenthal 2009] T. D. Browning and U. Derenthal, "Manin's conjecture for a cubic surface with $D_{5}$ singularity", Int. Math. Res. Not. 2009:14 (2009), 2620-2647. MR 2011a:14041 Zbl 1173.14017

[Chambert-Loir and Tschinkel 2002] A. Chambert-Loir and Y. Tschinkel, "On the distribution of points of bounded height on equivariant compactifications of vector groups", Invent. Math. 148:2 (2002), 421-452. MR 2003d:11094 Zbl 1067.11036

[Colliot-Thélène and Sansuc 1976] J.-L. Colliot-Thélène and J.-J. Sansuc, "Torseurs sous des groupes de type multiplicatif; applications à l'étude des points rationnels de certaines variétés algébriques", C. R. Acad. Sci. Paris Sér. A-B 282:18 (1976), A1113-A1116. MR 54 \#2657 Zbl 0337.14014 
[Colliot-Thélène and Sansuc 1980] J.-L. Colliot-Thélène and J.-J. Sansuc, "La descente sur les variétés rationnelles", pp. 223-237 in Journées de Géometrie Algébrique d'Angers (Angers, 1979), edited by A. Beauville, Sijthoff \& Noordhoff, Alphen aan den Rijn, Germantown, MD, 1980. MR 82d:14016 Zbl 0451.14018

[Colliot-Thélène and Sansuc 1987] J.-L. Colliot-Thélène and J.-J. Sansuc, "La descente sur les variétés rationnelles, II”, Duke Math. J. 54:2 (1987), 375-492. MR 89f:11082 Zbl 0659.14028

[Coray and Tsfasman 1988] D. F. Coray and M. A. Tsfasman, "Arithmetic on singular Del Pezzo surfaces”, Proc. London Math. Soc. (3) 57:1 (1988), 25-87. MR 89f:11083 Zbl 0653.14018

[Derenthal 2009] U. Derenthal, "Counting integral points on universal torsors", Int. Math. Res. Not. 2009:14 (2009), 2648-2699. MR 2010f:14019 Zbl 1173.14014

[Derenthal 2014] U. Derenthal, "Singular del Pezzo surfaces whose universal torsors are hypersurfaces”, Proc. Lond. Math. Soc. (3) 108:3 (2014), 638-681. MR 3180592 Zbl 1292.14027

[Derenthal and Loughran 2010] U. Derenthal and D. Loughran, "Singular del Pezzo surfaces that are equivariant compactifications", Zap. Nauchn. Sem. S.-Peterburg. Otdel. Mat. Inst. Steklov. (POMI) 377:10 (2010), 26-43. MR 2012b:14068 Zbl 06246152

[Derenthal et al. 2008] U. Derenthal, M. Joyce, and Z. Teitler, "The nef cone volume of generalized del Pezzo surfaces”, Algebra Number Theory 2:2 (2008), 157-182. MR 2009b:14069 Zbl 1158.14032

[Franke et al. 1989] J. Franke, Y. I. Manin, and Y. Tschinkel, "Rational points of bounded height on Fano varieties", Invent. Math. 95:2 (1989), 421-435. MR 89m:11060 Zbl 0674.14012

[Franz 2009] M. Franz, Convex - a Maple package for convex geometry, version 1.1, 2009, Available at http://www.math.uwo.ca/ mfranz/convex/.

[Hassett and Tschinkel 2004] B. Hassett and Y. Tschinkel, "Universal torsors and Cox rings", pp. 149-173 in Arithmetic of higher-dimensional algebraic varieties (Palo Alto, CA, 2002), edited by B. Poonen and Y. Tschinkel, Progr. Math. 226, Birkhäuser, Boston, 2004. MR 2005a:14049 Zbl 1077.14046

[Heath-Brown 1984] D. R. Heath-Brown, "Diophantine approximation with square-free numbers", Math. Z. 187:3 (1984), 335-344. MR 85j:11072 Zbl 0539.10026

[Heath-Brown 1997] D. R. Heath-Brown, "The density of rational points on cubic surfaces", Acta Arith. 79:1 (1997), 17-30. MR 98h:11083 Zbl 0863.11021

[Heath-Brown 2003] D. R. Heath-Brown, “The density of rational points on Cayley's cubic surface”, pp. 33 in Proceedings of the Session in Analytic Number Theory and Diophantine Equations (Bonn, 2002), edited by D. R. Heath-Brown and B. Z. Moroz, Bonner Math. Schriften 360, Univ. Bonn, Bonn, 2003. Zbl 1060.11038

[Le Boudec 2012a] P. Le Boudec, "Manin's conjecture for a cubic surface with $2 \mathrm{~A}_{2}+\mathrm{A}_{1}$ singularity type”, Math. Proc. Cambridge Philos. Soc. 153:3 (2012), 419-455. MR 2990624 Zbl 1253.14022

[Le Boudec 2012b] P. Le Boudec, "Manin's conjecture for two quartic del Pezzo surfaces with $3 \mathbf{A}_{1}$ and $\mathbf{A}_{1}+\mathbf{A}_{2}$ singularity types", Acta Arith. 151:2 (2012), 109-163. MR 2012j:11135 Zbl 1248.11045

[Loughran 2010] D. Loughran, "Manin's conjecture for a singular sextic del Pezzo surface", J. Théor. Nombres Bordeaux 22:3 (2010), 675-701. MR 2012a:14048 Zbl 1258.14029

[Peyre 1995] E. Peyre, "Hauteurs et mesures de Tamagawa sur les variétés de Fano", Duke Math. J. 79:1 (1995), 101-218. MR 96h:11062 Zbl 0901.14025

[Peyre 1998] E. Peyre, "Terme principal de la fonction zêta des hauteurs et torseurs universels", pp. 259-298 in Nombre et répartition de points de hauteur bornée (Paris, 1996), edited by E. Peyre, Astérisque 251, Société Mathématique de France, Paris, 1998. MR 2000f:11081 Zbl 0966.14016 
[Salberger 1998] P. Salberger, "Tamagawa measures on universal torsors and points of bounded height on Fano varieties”, pp. 91-258 in Nombre et répartition de points de hauteur bornée (Paris, 1996), edited by E. Peyre, Astérisque 251, Société Mathématique de France, Paris, 1998. MR 2000d:11091 Zbl 0959.14007

[Tanimoto and Tschinkel 2012] S. Tanimoto and Y. Tschinkel, "Height zeta functions of equivariant compactifications of semi-direct products of algebraic groups", pp. 119-157 in Zeta functions in algebra and geometry, edited by A. Campillo et al., Contemp. Math. 566, Amer. Math. Soc., Providence, RI, 2012. MR 2858922 Zbl 06092111

Communicated by Roger Heath-Brown

Received 2013-10-30 Revised 2014-03-05 Accepted 2014-04-26

pierre.leboudec@epfl.ch École Polytechnique Fédérale de Lausanne, SB MATHGEOM TAN, Bâtiment MA, Station 8, CH-1015 Lausanne, Switzerland 


\section{Algebra \& Number Theory}

msp.org/ant

\section{EDITORS}

MANAGING EDITOR

Bjorn Poonen

Massachusetts Institute of Technology

Cambridge, USA

\author{
EDITORIAL BOARD CHAIR \\ David Eisenbud \\ University of California \\ Berkeley, USA
}

BOARD OF EDITORS

Georgia Benkart

Dave Benson

Richard E. Borcherds

John H. Coates

J-L. Colliot-Thélène

Brian D. Conrad

Hélène Esnault

Hubert Flenner

Edward Frenkel

Andrew Granville

Joseph Gubeladze

Roger Heath-Brown

Craig Huneke

Yujiro Kawamata

János Kollár

Yuri Manin

Barry Mazur

Philippe Michel

Susan Montgomery
University of Wisconsin, Madison, USA

University of Aberdeen, Scotland

University of California, Berkeley, USA

University of Cambridge, UK

CNRS, Université Paris-Sud, France

University of Michigan, USA

Freie Universität Berlin, Germany

Ruhr-Universität, Germany

University of California, Berkeley, USA

Université de Montréal, Canada

San Francisco State University, USA

Oxford University, UK

University of Virginia, USA

University of Tokyo, Japan

Princeton University, USA

Northwestern University, USA

Harvard University, USA

École Polytechnique Fédérale de Lausanne

University of Southern California, USA
Shigefumi Mori

Raman Parimala

Jonathan Pila

Anand Pillay

Victor Reiner

Peter Sarnak

Joseph H. Silverman

Michael Singer

Vasudevan Srinivas

J. Toby Stafford

Bernd Sturmfels

Richard Taylor

Ravi Vakil

Michel van den Bergh

Marie-France Vignéras

Kei-Ichi Watanabe

Efim Zelmanov

Shou-Wu Zhang
RIMS, Kyoto University, Japan

Emory University, USA

University of Oxford, UK

University of Notre Dame, USA

University of Minnesota, USA

Princeton University, USA

Brown University, USA

North Carolina State University, USA

Tata Inst. of Fund. Research, India

University of Michigan, USA

University of California, Berkeley, USA

Harvard University, USA

Stanford University, USA

Hasselt University, Belgium

Université Paris VII, France

Nihon University, Japan

University of California, San Diego, USA

Princeton University, USA

\section{PRODUCTION}

production@msp.org

Silvio Levy, Scientific Editor

See inside back cover or msp.org/ant for submission instructions.

The subscription price for 2014 is US $\$ 225 /$ year for the electronic version, and $\$ 400 /$ year ( $\$ 55$, if shipping outside the US) for print and electronic. Subscriptions, requests for back issues and changes of subscribers address should be sent to MSP.

Algebra \& Number Theory (ISSN 1944-7833 electronic, 1937-0652 printed) at Mathematical Sciences Publishers, 798 Evans Hall \#3840, c/o University of California, Berkeley, CA 94720-3840 is published continuously online. Periodical rate postage paid at Berkeley, CA 94704, and additional mailing offices.

ANT peer review and production are managed by EditFLOw ${ }^{\circledR}$ from Mathematical Sciences Publishers.

\section{PUBLISHED BY}

mathematical sciences publishers

nonprofit scientific publishing

http://msp.org/

(C) 2014 Mathematical Sciences Publishers 


\section{Algebra \& Number Theory}

Volume $8 \quad$ No. $5 \quad 2014$

Polarization estimates for abelian varieties

DAVID MASSER and GISBERT WÜSTHOLZ

Compatibility between Satake and Bernstein isomorphisms in characteristic $p$

1071

RACHEL OLLIVIER

The final $\log$ canonical model of $\bar{M}_{6}$

1113

FABIAN MÜLLER

Poisson structures and star products on quasimodular forms

1127

FRANÇOIS DUMAS and EMMANUEL ROYER

Affinity of Cherednik algebras on projective space

GWYN BELLAMY and MAURIZIO MARTINO

ALEXANDRU CHIRVASITU

Tetrahedral elliptic curves and the local-global principle for isogenies

1201

BARINDER S. BANWAIT and JOHN E. CREMONA

Local cohomology with support in generic determinantal ideals

1231

Claudiu RAICU and JERZY WEYMAN

Affine congruences and rational points on a certain cubic surface

1259

PIERRE LE BoudeC 